\title{
Real World Assessment of an Auto-parametric Electromagnetic Vibration Energy Harvester
}

\author{
Yu Jia ${ }^{1,2, *}$, Jize Yan $^{3}$, Sijun Du ${ }^{1}$, Tao Feng ${ }^{1}$, Paul Fidler ${ }^{1}$, \\ Campbell Middleton ${ }^{1}$, Kenichi Soga ${ }^{1,4}$ and Ashwin A. Seshia ${ }^{1,+}$ \\ ${ }^{1}$ Department of Engineering, University of Cambridge, Cambridge CB2 1PZ, UK \\ ${ }^{2}$ Department of Mechanical Engineering, University of Chester, Chester CH2 4NU, UK \\ ${ }^{3}$ School of Electronics and Computer Science, University of Southampton, Southampton SO17 1BJ, UK \\ ${ }^{4}$ University of California-Berkeley, Berkeley, CA, USA \\ E-mail: *yu.jia.gb@ieee.org, ${ }^{+}$aas41@cam.ac.uk
}

Draft: August 3, 2017

\begin{abstract}
The convention within the field of vibration energy harvesting (VEH) has revolved around designing resonators with natural frequencies that match single fixed frequency sinusoidal input. However, real world vibrations can be random, multi-frequency, broadband and timevarying in nature. Building upon previous work on auto-parametric resonance, the fundamentally different approach allows multiple axes vibration and has the potential to achieve higher power density as well as wider operational frequency bandwidth. This paper presents the power response of a packaged auto-parametric VEH prototype (practical operational volume $\sim 126 \mathrm{~cm}^{-3}$ ) towards various real world vibration sources including vibration of a bridge, a compressor motor as well as an automobile. At auto-parametric resonance (driven at $23.5 \mathrm{~Hz}$ and $1 \mathrm{~g}_{r m s}$ ), the prototype can output a peak of $78.9 \mathrm{~mW}$ and $4.5 \mathrm{~Hz}$ of $-3 \mathrm{~dB}$ bandwidth. Furthermore, up to $\sim 1 \mathrm{~mW}$ of average power output was observed from the harvester on the Forth Road Bridge. The harvested electrical energy from various real world sources were used to power up a power conditioning circuit, a wireless sensor mote, a MEMS (micro-electromechanical system) accelerometer and other low power sensors. This demonstrates the concept of self-sustaining vibration-powered wireless sensor systems in real world scenarios, to potentially realise maintenance-free autonomous structural health and condition monitoring.
\end{abstract}

Keywords: electromagnetic, parametric resonance, vibration energy harvesting, real vibration data, bridge, compressor, automobile

\section{Introduction}

Vibration energy harvesting (VEH) is an emerging technology that harnesses ambient kinetic energy such as the vibration of machines, passing traffic, human motion, etc., in an attempt to power micro-electronic systems in a decentralised and self-sustaining manner (Priya and Inman, 2009; Kazmierski and Beeby, 2011). One of the key motivation for $\mathrm{VEH}$ is the potential to realise net-zero-power wireless sensor networks (WSN), which would in turn enable maintenance-free structural health monitoring (SHM) and condition monitoring applications.

Most of the existing WSN systems for SHM rely on batteries, which require regular maintenance and replacement (Beeby et al., 2006). Battery servicing can amount to non-trivial costs (Priya and Inman, 2009; Beeby and White, 2010) due to skilled labour, logistics and carbon footprint of battery servicing. This battery maintenance cost becomes even higher when dealing with: vast civil infrastructural or industrial structures such as water and gas distribution systems and oil drilling wells, difficult-to-access areas such as buried or embedded systems, locations that require the erection of temporary access structures such as scaffolds, potential disruption to services such as rail and transportation applications, and potentially hazardous environments such as off-shore or nuclear applications.

VEH attempts to address these issues by providing an on-board and self-replenishing power solution for the micro-electronic systems (Kazmierski and Beeby, 2011). However, overwhelming majority of 
reported VEH to date relies on matching the natural frequency of the mechanical resonator to a sinusoidal frequency source rather than representative real vibration data (Priya and Inman, 2009; Beeby et al., 2006; Mitcheson et al., 2008). Real world vibration can be significantly more sophisticated than a single frequency sine wave and can potentially be random, multi-frequency, broadband and time-varying in nature. Therefore, the single frequency direct resonant approach are typically inadequate when faced with real vibration.

There has been various attempts in the literature to address the bandwidth issue of VEH, such as: active frequency tuning by varying system parameters such as stiffness or dimensions (Hu et al., 2007; Challa et al., 2011; Weddell et al., 2012), passive frequency tuning by various shunt capacitance (Muriuki, 2004), arraying either uncoupled (Shahruz, 2006; Feng and Hung, 2007; Xue et al., 2008) or coupled (Petropoulos et al., 2004; Wong et al., 2009) oscillators of varying dimensions to capture a broader bandwidth, use of mechanical stoppers to induce nonlinear amplitude flattening (Soliman et al., 2008, 2009), nonlinear oscillators (Burrow and Clare, 2007; Mann and Sims, 2009; Ramlan et al., 2010), and multi-stability (Stanton et al., 2010; Zhu and Beeby, 2013; Trigona et al., 2013).

Previous work from our group has explored a fundamentally different resonant approach, namely parametric resonance (Jia et al., 2013c). When compared with the conventional direct resonance, it has the potential to attain higher power output (Jia et al., 2013c,b), wider operational frequency bandwidth (Jia et al., 2013c,b; Jia and Seshia, 2013) and access multiple frequency windows at higher orders of this alternative vibration phenomenon (Jia et al., 2013a). These have been numerically and experimentally demonstrated at both macro-scales (Jia et al., 2013c, 2014) as well as MEMS (micro-electromechanical system) scales (Jia et al., 2013b,a). Additionally, the coupling of both direct and parametric resonance have been explored to realise multi-resonant regime harvesting (Jia and Seshia, 2013) and the principle of auto-parametric resonance has also been realised (Jia and Seshia, 2014) to access the more profitable regions of parametric resonance at lower acceleration levels.

Equations 1 and 2 summarise the governing equations of a direct oscillator coupled to a parametric oscillator (Jia and Seshia, 2014), which forms a type of auto-parametric oscillator. The direct oscillator posses a direct forcing term on the right hand side of the equation. On the other hand, the parametric oscillator consists of a time coefficient in its system parameter, representing the parametric modulation as a function of time induced by the response of the direct oscillator.

$$
\begin{array}{r}
\ddot{x}+2 \zeta_{x 1} \omega_{x n} \dot{x}+\zeta_{x 2} \dot{x}|\dot{x}|+\omega_{x n}^{2} x+\mu_{x} x^{3}-\psi\left(\dot{y}^{2}+y \ddot{y}\right)=a \cos \omega t+W(t) \\
\ddot{y}+2 \zeta_{y 1} \omega_{y n} \dot{y}+\zeta_{y 2} \dot{y}|\dot{y}|+\omega_{y n}^{2} y+\mu_{y} y^{3}-\xi \ddot{x}(t) y=0
\end{array}
$$

where, $x$ and $y$ are the response displacement, $\zeta_{x 1}$ and $\zeta_{y 1}$ are the viscous damping factors, $\zeta_{x 2}$ and $\zeta y 2$ are the nonlinear quadratic damping terms, $\mu_{x}$ and $\mu_{y}$ are the Duffing coefficients, $\psi$ is the backward coupling term, $\xi$ is the parametric mode coupling term, $a$ is the external acceleration amplitude, $\omega$ is the excitation frequency, $W(t)$ is external noise vibration, $t$ is the time domain, and $\omega_{x n}$ and $\omega_{y n}$ are the natural frequencies of the direct and parametric oscillators respectively.

An auto-parametric resonator has both a directly excited component, which converts external forcing into an internal parametric excitation for its parametrically excited component. The resultant autoparametric harvester device is responsive to the conventional directly excited resonance, but also possess the potential to operate in the potentially more profitable parametric resonance regimes when the conditions become favourable as illustrated in figure 1 .

Across the wide range of directly excited, parametrically excited and other nonlinear VEH systems, there has been extensive work in the reported literature on the design, optimisation and testing of electromagnetic (Priya and Inman, 2009; Beeby and White, 2010; Spreemann and Manoli, 2012) and piezoelectric VEH (Priya and Inman, 2009; Erturk and Inman, 2011; Montanini and Quattrocchi, 2016) devices supposedly targeted at various applications around the 10's $\mathrm{Hz}$ to 100's $\mathrm{Hz}$ range. However, overwhelming majority of these devices are optimised and characterised using single source sinusoidal excitation under controlled laboratory conditions. In real world applications, vibration sources have unpredictable characteristics including possibilities for sources to demonstrate random time-varying, noisy or broadband characteristics. Even for relatively fixed frequency sources, such as that produced by a fixed speed engine (Weddell et al., 2012), the signal is rarely purely sinusoidal but instead superimposed with a noisy background excitation.

While strides have been made in the design and optimisation of VEH (Arroyo and Badel, 2011), published studies on the real world application validation and performance assessment are limited. Existing studies include the analysis of measured vibration in order to feed into modelling and design optimisation (Green et al., 2013), field trial evaluation of a macro-scale simple cantilever piezoelectric 

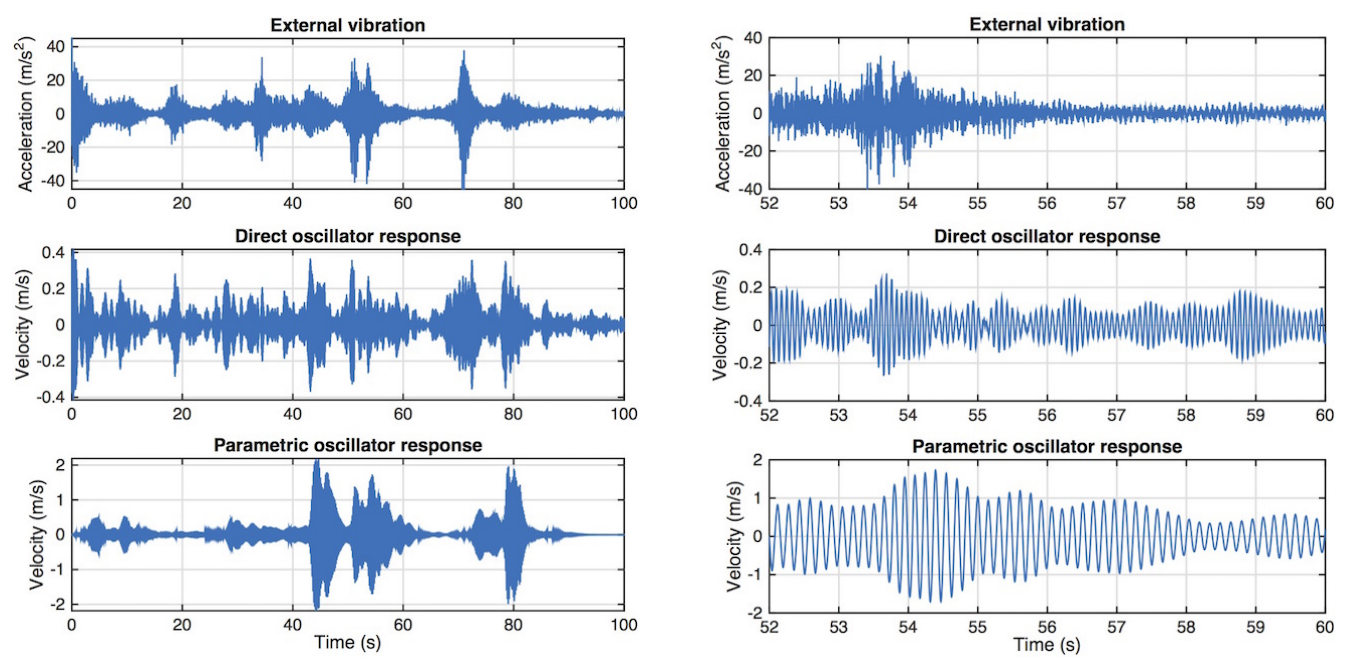

(a) Time domain

(b) Zoomed in view

Figure 1: Numerical simulation of an auto-parametric oscillator when subjected to measured real bridge vibration. The zoomed in view shows the 2:1 ratio between the response frequencies of the direct oscillator and the parametric oscillator. When condition becomes favourable, the more superior parametric resonance onsets.

device that is limited to 10's $\mu \mathrm{W}$ output range (Peigney and Siegert, 2013), and field trial testing of a meso-scale optimised directly excited design that delivers sub-microwatt power output when operating in real world vibration environment (Galchev et al., 2011). A key challenge for most VEH devices tested in real-world environments is the low voltage output achievable, limiting the practical viability as a useful power sources. Furthermore, the low voltage amplitudes under these conditions results in sub-optimal energy conversion efficiencies due to diode thresholds and leakage currents associated with the power conditioning circuits.

This study joins in with the few reported studies of real-world field trial experimentation of the VEH technology in general(Peigney and Siegert, 2013; Galchev et al., 2011), and is the first study of this kind to validate the feasibility and assess the performance of $\mathrm{VEH}$ based on the parametric resonant principle. A key element is to assess the wider frequency band performance of parametric resonant $\mathrm{VEH}$, in contrast to directly excited systems. Packaged prototypes were designed based on previously reported proof-of-concept devices (Jia and Seshia, 2014). The packaged auto-parametric resonant VEH presented here employs a similar electromagnetic transducer reported in another previous study (Jia et al., 2013c).

A variety of real world vibration data were collected, including from a bridge, a compressor motor and an automobile. The frequency characteristics of these vibration sources were analysed, along with the power response of the packaged auto-parametric VEH prototype towards these various sources. Prototypes were subjected to vibration from both real-data-programmed shaker as well as real site trials. Additionally, the recovered power by the prototype from these real vibration were used to power a commercial power conditioning circuit, a wireless sensor mote and a variety of commercial low power sensors. This helped to experimentally demonstrate an end-to-end self-powered WSN system solution based on harvesting real and representative vibration sources.

\section{Harvester prototype and WSN setup}

\section{Vibration energy harvester}

The harvester prototype developed was designed based on the previously reported piezoelectric autoparametric resonator (Jia and Seshia, 2014). However, in this instance, an electromagnetic transducer was employed using neodymium iron boron magnets and a copper disk coil with wire resistance of $\sim 4 \mathrm{k} \Omega$ based another previous work (Jia et al., 2013c). The coil is fixed at the centre, while magnets attached to the tip of the auto-parametric resonator can move and cut through the magnetic flux lines.

The packaged device shown in figure 2 a has a protection enclosure volume of $\sim 300 \mathrm{~cm}^{3}(10 \mathrm{~cm} \times 5$ $\mathrm{cm} \times 6 \mathrm{~cm})$, a practical operational device volume of $\sim 126 \mathrm{~cm}^{3}(9 \mathrm{~cm} \times 2.8 \mathrm{~cm} \times 5 \mathrm{~cm})$ and an active component volume of $<50 \mathrm{~cm}^{3}$. Additionally, manual mechanical adjustments were designed into the 


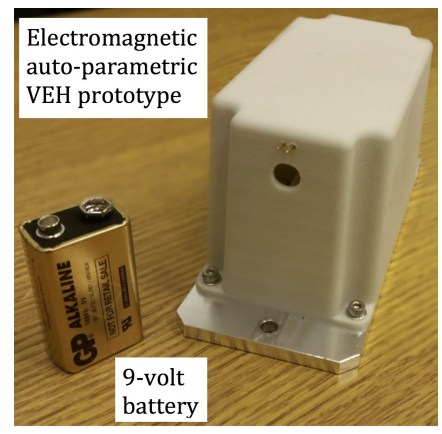

(a) Prototype device compared to a 9 -volt battery

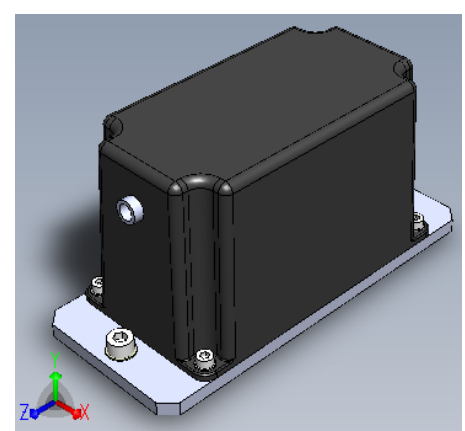

(b) Isometric perspective view of the packaged CAD prototype.

Figure 2: The packaged electromagnetic auto-parametric vibration energy harvester prototype.

prototype to allow minor frequency tuning, which could be eventually replaced by permanent fixtures for further miniaturisation. The auto-parametric resonator was designed and tuned to a natural frequency of $\sim 13.2 \mathrm{~Hz}$. The directly excited initial spring structure discussed in (Jia et al., 2014) was designed to approximately twice this frequency in order to provide resonant-based amplification of the parametric excitation, which enabled auto-parametric resonance as shown in (Jia and Seshia, 2014).

The experimental setup within the lab are shown in figure 3, where the VEH prototype rests on a vibration shaker. The shaker is programmed by a function/arbitrary generator via a power amplifier, in order to assess the prototype response when subjected to fixed frequencies, frequency sweeps and bandlimited white noise. Measured real vibration data were sent from a computer to the arbitrary waveform generator of the function generator. The amplitude of the arbitrary waveform (measured vibration) was then matched to the measured acceleration amplitude using an accelerometer mounted on the shaker. Output from either the harvester (raw AC signal) or from the power conditioning circuit (conditioned power of the harvester output), as well as storage capacitors and signals from WSN mote or sensors were monitored on a digital oscilloscope.

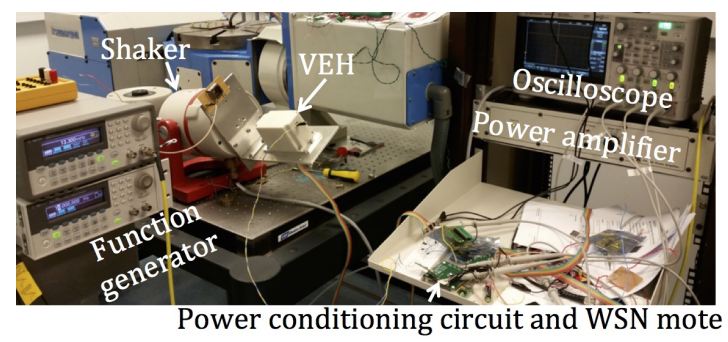

Figure 3: Experimental setup within the lab.

When a vertical excitation at approximately twice the natural frequency is subjected to the prototype, parametric resonance can be activated as illustrated in figure 4 . Unlike directly forced oscillations, where the response frequency matches the excitation, parametric resonant response is always at around the natural frequency. Therefore, the characteristics of the principal (first order) parametric resonance is when the response frequency is half of the excitation frequency.

The matched load resistance for direct resonant response is in the range of $4 \mathrm{k} \Omega$ to $5 \mathrm{k} \Omega$, at which maximum power can be extracted from the electromagnetic transducer. The power characteristics at varying load resistance when driven into parametric resonance is shown in figure 5 . Due to the presence of a damping-dependent initiation threshold amplitude, the matched load resistance varies more noticeably. At larger parallel load resistance, the electrical damping is lower and parametric resonance onsets at lower acceleration levels. However, at the presence of high drive acceleration, a low parallel load resistance (high electrical damping) can be afforded in order to access higher electrical power output.

Figure 6 summaries the power values at varying acceleration levels for the harvester prototype when operated in direct and parametric resonant regimes. These power performance are also contrasted with a commercial electromagnetic vibration energy harvester of comparable size: Perpetuum PMG-17 (Perpetuum, 2017), which operates in direct resonance. The initiation threshold amplitude of parametric resonance is $\sim 0.18 \mathrm{~g}_{r m s}$, after which, the parametric resonant operation outperforms its direct resonant 

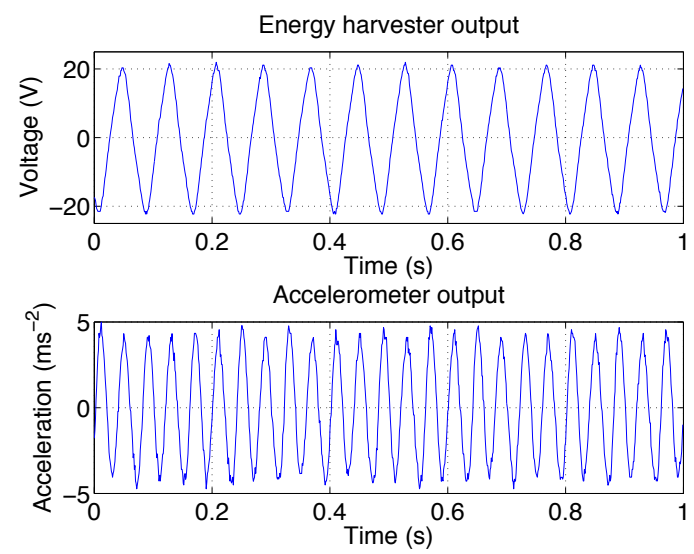

Figure 4: The harvester prototype driven into principal parametric resonance. The response frequency from the harvester is in the vicinity of the natural frequency and is half that of the excitation frequency.

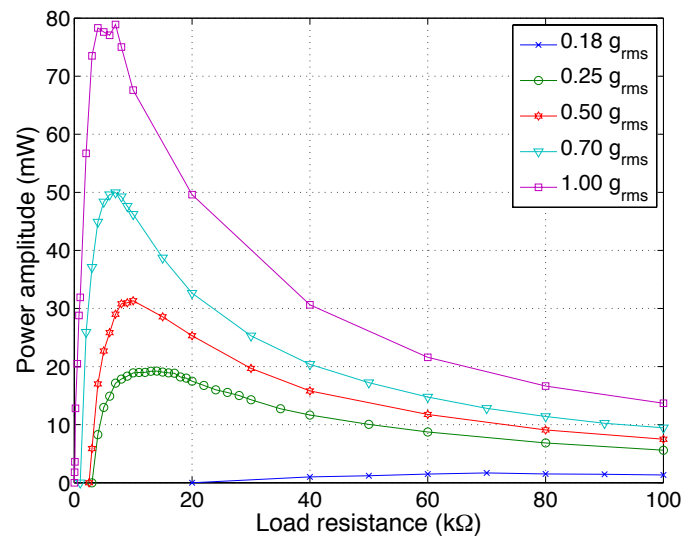

Figure 5: Parametric resonant power characteristics under varying load resistance and acceleration levels.

counterparts. Note that the electromagnetic transducer used in the current test prototype is still far from fully optimised, compared to the existing commercial solution.

PMG-17 operates in the range of $\sim 120 \mathrm{~Hz}$, has a half power bandwidth of $\sim 15 \mathrm{~Hz}(12.5 \%)$ of the resonant frequency $f_{r}$ ) and can deliver a maximum of $\sim 45 \mathrm{~mW}$ at $1 \mathrm{~g}_{r m s}$ of excitation. On the other hand, at the same level of acceleration, the prototype reported here has a frequency characteristics shown in figure 7 . Direct resonance at $13.2 \mathrm{~Hz}$ recorded $\sim 2 \mathrm{~Hz}$ of half power band (15.2\%), while parametric resonance exhibited up to $\sim 4.5 \mathrm{~Hz}(19.2 \%)$ of half power band at approximately twice the natural frequency. Table 1 summaries the performance of PMG-17 and the auto-parametric prototype. The metric employed for the comparison are two of the most widely used from the literature; namely, power density normalised against acceleration squared (N.P.D) (Priya and Inman, 2009; Beeby et al., 2006) and a figure of merit derived from N.P.D normalised against operational bandwidth by resonant frequency (F.O.M) (Mitcheson et al., 2008; Andosca et al., 2012).

Table 1: Comparison of the power performance of the reported auto-parametric prototype when driven into direct resonance and parametric resonance, and a commercial VEH (Perpetuum, 2017). All are subjected to 1.0 $\mathrm{g}_{r m s}\left(13.9 \mathrm{~ms}^{-2}\right)$ of acceleration at their resonant frequencies. H.P.B. denotes half power bandwidth, N.P.D. denotes normalised power density (Priya and Inman, 2009; Beeby et al., 2006) and F.O.M. represents figure of merit (Mitcheson et al., 2008; Andosca et al., 2012).

\begin{tabular}{|c|c|c|c|c|c|c|c|c|}
\hline Device & $\begin{array}{c}\text { Operational } \\
\text { Mode at } f_{r}(\mathrm{~Hz})\end{array}$ & $\begin{array}{l}\text { Power } \\
(\mathrm{mW})\end{array}$ & $\begin{array}{c}\text { H.P.B. } \\
(\mathrm{Hz})\end{array}$ & $\begin{array}{l}\text { Vol. } \\
\left(\mathrm{cm}^{3}\right)\end{array}$ & $\begin{array}{l}\text { H.P.B. } / f_{r} \\
\quad(\%)\end{array}$ & $\underset{(\%)}{\sum \text { H.P.B. } / f_{r}}$ & $\begin{array}{l}\text { N.P.D. } \\
(\mu \mathrm{Wcm}\end{array}$ & $\begin{array}{l}\text { F.O.M. } \\
\left.\mathrm{m}^{-2} \mathrm{~s}^{4}\right)\end{array}$ \\
\hline \multirow{2}{*}{ This device } & Parametric (23.5) & 78.9 & 4.5 & 126 & 19.2 & \multirow{2}{*}{34.3} & 3.25 & 0.62 \\
\hline & Direct (13.2) & 64.8 & 2.0 & 126 & 15.2 & & 2.67 & 0.40 \\
\hline PMG-17 & Direct (120) & 45 & 15 & 135 & \multicolumn{2}{|r|}{12.5} & 1.73 & 0.22 \\
\hline
\end{tabular}




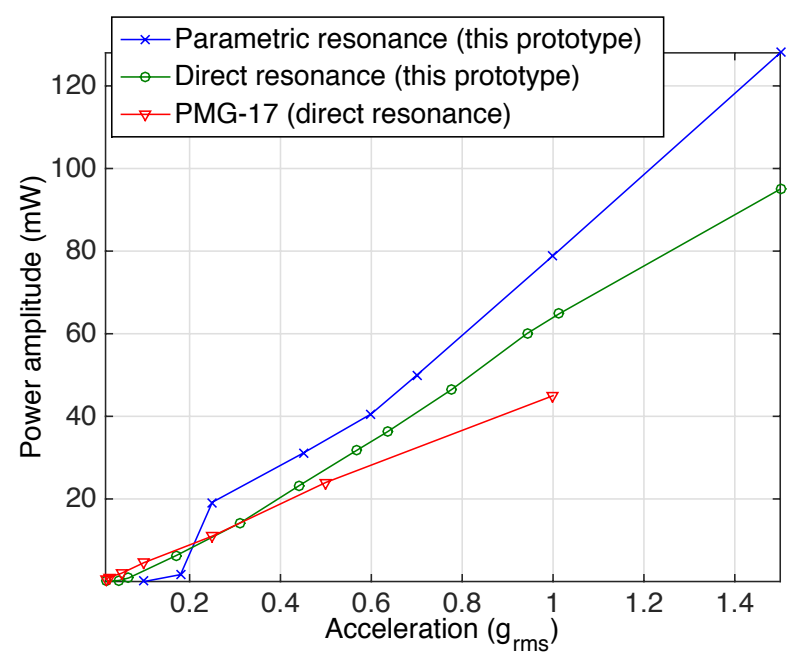

Figure 6: Power response per acceleration for the prototype $\left(\sim 126 \mathrm{~cm}^{3}\right)$ when driven into parametric resonance $(\sim 25 \mathrm{~Hz})$ and direct resonance $(13 \mathrm{~Hz})$; compared to the power performance of Perpetuum PMG-17 (Perpetuum, 2017) $\left(\sim 135 \mathrm{~cm}^{3}, 100 \mathrm{~Hz}\right)$.

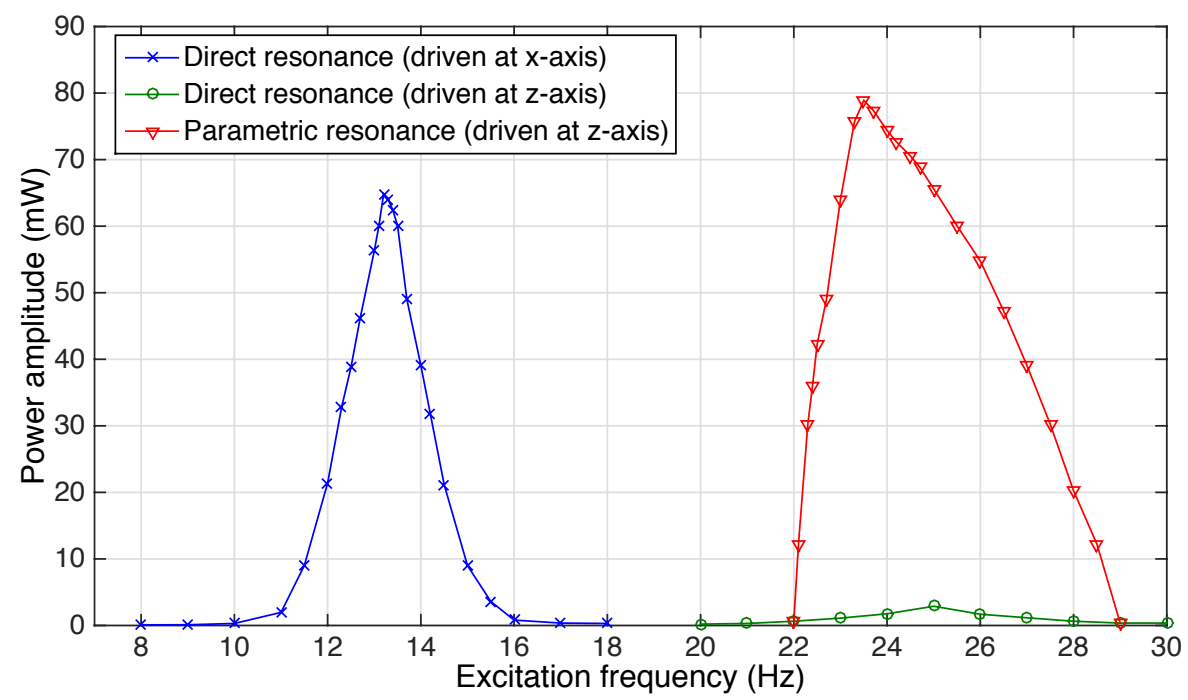

Figure 7: Frequency domain power response driven at $1.0 \mathrm{~g}_{r m s}$. The fundamental mode direct resonance has a half power bandwidth of $\sim 2 \mathrm{~Hz}$ and the first order parametric resonance has a half power bandwidth of $\sim 4.5 \mathrm{~Hz}$.

The peak N.P.D. of the reported auto-parametric prototype is nearly twice as high, and F.O.M. thrice as high as the comparable existing commercial device when subjected to the same level of acceleration. Furthermore, the accumulative relative half power bandwidth (H.P.B.) per resonant frequency $f_{r}$, represented by: $\left(\sum\right.$ H.P.B. $\left./ f_{r}\right)$, is nearly 3 times higher.

\section{Power conditioning circuit and kit setup}

For integration with a WSN mote and sensor system, a commercial power conditioning board (LTC35881 from Linear Technology) was employed to rectify, store and regulate the harvested power. A $5 \mathrm{mF}$ (experimentally measured) supercapacitor (AVX BestCap) was added to the storage of the circuit. This power conditioning board from Linear Technology consists of a conventional diode bridge rectifier and a buck converter circuit. The input voltage of the harvester is very demanding due to the need to both overcome the threshold of the diode bridge and exceed the voltage across the storage capacitor.

Therefore, a passive voltage multiplier in the form of a 6 stage Dickson charge pump circuit was employed to amplify the voltage signal from the harvester prior to feeding into the power conditioning board. While this renders the rectifier circuit in LTC3588-1 redundant, the voltage regulation and switching options from the power conditioning board were still relevant. The diodes and capacitors 
employed for the charge pump circuit (assembled on an in-house fabricated printed circuit board) were Diodes Inc. IN5711WS-7-F Schottky diode and $47 \mu \mathrm{F}$ AVX Multilayer Ceramic Capacitor respectively.

Either $1.8 \mathrm{~V}_{d c}, 2.5 \mathrm{~V}_{d c}$ or $3.3 \mathrm{~V}_{d c}$ regulated DC supply from the power conditioning board was selected for the various systems depending on the required voltage. The regulated DC supply switches on when the voltage across the storage capacitor $\left(\mathrm{V}_{c a p}\right)$ attains $4.0 \mathrm{~V}$ (during charging) and remains on until $\mathrm{V}_{\text {cap }}$ drops below $3.0 \mathrm{~V}$ (for discharging); thus, creating an energy buffer to avoid pre-mature 'switch off' from the initial turn-on power drain of the electrical load.

Therefore, depending on the capacitance of the power storage, the size of this energy buffer can be varied. In this instance, the energy buffer was $\sim 17.5 \mathrm{~mJ}$. A net positive input of conditioned power into the storage capacitor, after the turn on of the regulated voltage (power drain from a load), enables further accumulation of energy in the storage as $V_{c a p}$ continues to increase until $20.0 \mathrm{~V}$. This then, in the present configuration, corresponds to a maximum of $\sim 0.98 \mathrm{~J}$ stored electrical energy available for load consumption.

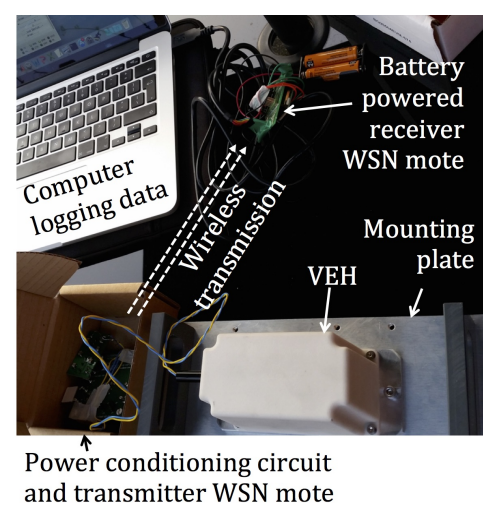

(a) Setup

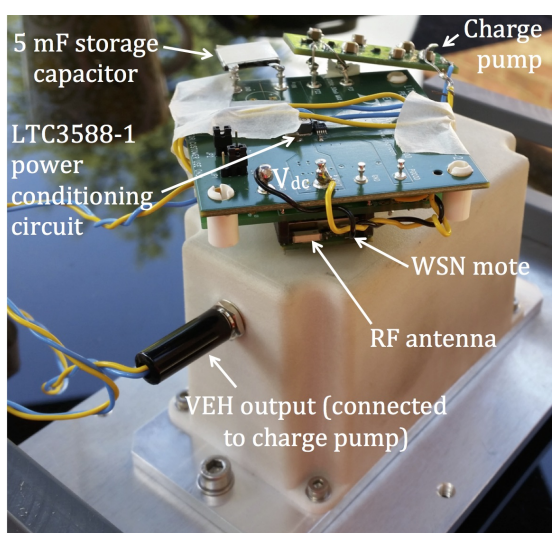

(b) kit

Figure 8: Prototype and test kit setup for site trial.

Figure 8 illustrates a particular kit setup that was used for various site trial testing. The VEH prototype is mechanically mounted on a mounting plate, which has a magnetic block on the bottom of either sides to enable magnetic attachment to various structures for SHM. The VEH is electrically connected to the AC input terminals of the LTC3588-1 power conditioning circuit, via a 6 stage voltage multiplier circuit (Dickson charge pump configuration). The charge pump circuit was included to promote the voltage amplitude for charging the $\mathrm{V}_{c a p}$ to at least the $4.0 \mathrm{~V}$, which is required to turn on $\mathrm{V}_{d c}$.

\section{Wireless sensor mote}

A WSN mote, based on the IEEE 802.15.4 standard, was connected to $\mathrm{V}_{d c}$. This WSN standard is designed for low power and low speed wireless communication. It is widely applied in media access and supports low latency wireless communication between devices equipped with compatible transceivers (Gutierrez et al., 2011).

The WSN mote (figure 9) in the demonstration was developed based on Atmel Lightweight Mesh (LWM). LWM is a low power wireless mesh protocol built on top of IEEE802.15.4 standard. According to the LWM specification, the devices in the network can be categorised into three types including coordinator, router and end-device. The coordinator is responsible for network maintenance and data aggregation. The router is used for routing and forwarding network message to the specified target. The end-device can be used for implementing low power operation (Atmel Corporation, 2015).

LWM has better performance and smaller footprint than Atmel BitCloud (Cervenka et al., 2014). Also, in comparison to other WSN systems such as Contiki, the implementation of putting devices into deep sleep mode in LWM is more explicitly achievable. Therefore, LWM is ideal for developing robust and low power WSN system in resource constrained devices.

Each mote is equipped with ATMega128rfa1 micro-controller with IEEE 802.15.4 compliant $2.4 \mathrm{GHz}$ $\mathrm{RF}$ transceiver. The sensor motes also provide interfaces for integration of different types of sensors such as tilt sensor, displacement sensor, accelerometer, temperature sensor or other sensors applying supported peripheral device communication standard. 


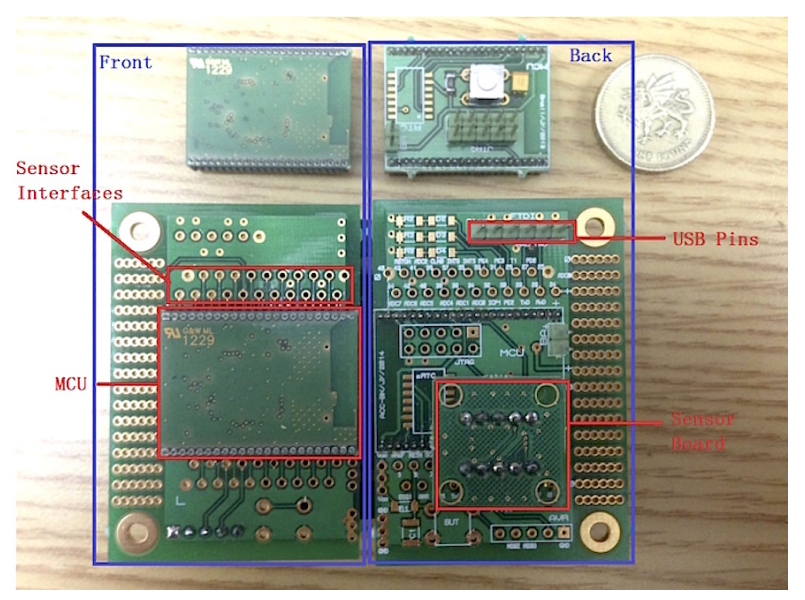

Figure 9: Wireless sensor mote used in the demonstration.

It is shown in figure 9 that there are two types of sensor motes in different sizes available for different applications. The mote in larger size can be used for developing and prototyping sensing devices by integrating different sensors to the sensor mote using the existing interfaces without the effort of remanufacturing PCBs. The mote in smaller size can be used for making compacted sensing devices.

During the demonstration and trial tests, the smaller motes where employed for integration with the harvester. The network structure included: an End-device mote powered by a vibration energy harvesting (VEH) device and a Coordinator mote powered by battery. A computer terminal was further used to log data from the Coordinator via USB-to-Serial cable.

To ensure ultra-low power operation, the End-device mote was programmed to observe the algorithm outlined in figure 10. Therefore, the End-device mote does not expend extra power for network maintenance and the sampling/transmission rate was pre-programmed to a pre-defined interval (10 seconds or 60 seconds during the demonstration tests).

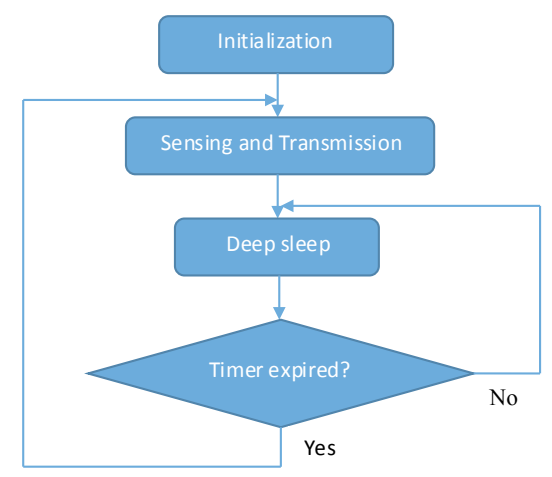

Figure 10: Flowchart summarising the algorithm of the End-device mote.

The battery-powered Coordinator mote on the other hand, as recommended in the LWM specification, was always powered on to receive and process incoming packets. This WSN configuration is suitable for relatively small area of network in applications such as automobile and compressor/engine monitoring, where the receiver can be connected to an energy abundant source such as car battery or mains supply. Even in certain infrastructural applications such as SHM of bridges, the receiver or gateway can be connected to various mains power outlets along the maintenance access or the mains supply typically available for road lighting, while the ultra-low power transmitter is attached to monitor hard-to-access structures below the deck.

Through monitoring the voltage drop across $\mathrm{V}_{c a p}$, various energy consumption and power budget values were calculated for the VEH-powered transmitter mote (End-device). The initial turn on of the WSN mote (initialisation, routing and first transmission), in the absence of sensors, required $\sim 7.6 \mathrm{~mJ}$ of energy as $\mathrm{V}_{\text {cap }}$ dropped from $4.0 \mathrm{~V}$ to $3.6 \mathrm{~V}$. Successive transmissions consumed $\sim 0.55 \mathrm{~mJ}$ each time. However, when communication with a receiver mote cannot be established, the transmitter typically consumes significantly more energy. Additionally, sleep mode and leakage from various electrical compo- 
nents total between $\sim 1.5 \mu \mathrm{W}$ to $\sim 3 \mu \mathrm{W}$ depending on the value of $\mathrm{V}_{\text {cap }}$. At larger $\mathrm{V}_{\text {cap }}$ values, leakage from the supercapacitor is higher. For $V_{\text {cap }}$ value in the range of $4.0 \mathrm{~V}$ and WSN mote duty cycling set to 1 transmission per minute, the average power consumption over 1 minute is $\sim 11 \mu \mathrm{W}$ (inclusive of 1 transmission, deep sleep mode operation and component leakage).

\section{Bridge Demonstration}

\section{Site trial test}

The site chosen for real bridge environment testing was the Forth Road Bridge. It is a $2.5 \mathrm{~km}$ long suspension bridge north west of Edinburgh city centre, connecting South Queensferry in Edinburgh and North Queensferry in Fife, over the Firth of Forth estuary. The suspension bridge was in operation since 1964 and has a main span of 1006 metres (Amey, 2017). On average, the road bridge services traffic in the order of 60 thousand to 70 thousand per day.

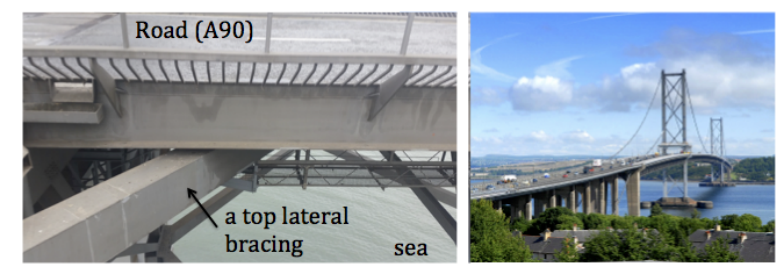

Figure 11: Forth Road Bridge.

All site testing measurements reported here were taken from around top lateral bracing 53 of the Forth Road Bridge (part of the main span) on the morning of 14 April 2015; and part of the early results were reported in PowerMEMS 2015 (Jia et al., 2015b). Figure 11 illustrates the location around which the tests were carried out. Ambient temperature was in the range of $14 \pm 3^{\circ} \mathrm{C}$ with relatively moderate wind conditions for the bridge ( $\sim 10 \mathrm{mph}$ to $\sim 15 \mathrm{mph}$ reported by Queensferry weather station). The speed limit for the bridge section of the A90 was $50 \mathrm{mph}(\sim 80 \mathrm{~km} / \mathrm{h})$ on the day (the normal speed limit for the bridge).

Lateral bracing and cross girder underneath the deck were identified as potential locations and structures for vibration powered structural health monitoring. This was due to the presence of non-trivial levels vibration as well as an interest to monitor the health of these repeatedly stressed critical support structures. A significant amount of vibration experienced by these structures were traffic induced. Therefore, the amplitude of vibration depends on the speed and size of the vehicle passing over these structures. For instance, lorries, trucks or heavy vehicles would typically result in more vigorous vibration.

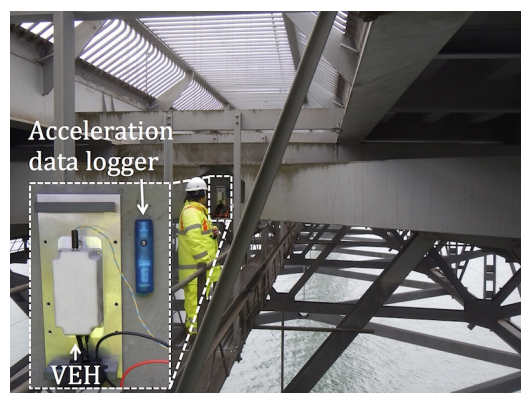

Figure 12: Vibration energy harvester (VEH) prototype attached to a cross girder on the Forth Road Bridge.

The two locations accessed and tested are shown in figures 12 (cross girder) and 13 (top lateral). The structures in this particular section of the bridge were accessible through the service and maintenance walkways underneath the deck. However, bottom lateral and several other locations of the bridge would require the erection of scaffolds and temporary access structures. Therefore, battery replacement for potential WSN systems to monitor these structures can be costly and time consuming.

As shown in figure 13, the VEH powered WSN mote was attached to both the side and top of the top lateral. Both the horizontal axis (orthogonal to the top lateral length) and the vertical axis exhibited 


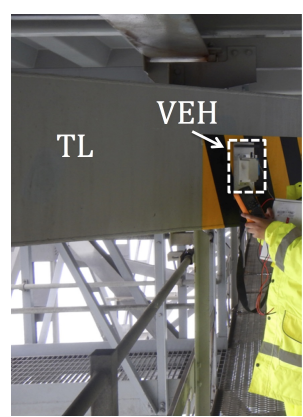

(a) Side mounted

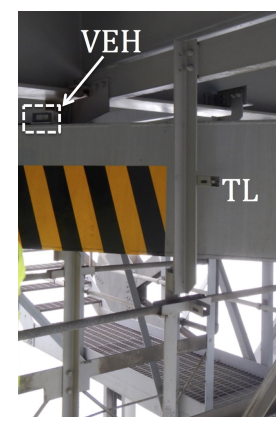

(b) Top mounted

Figure 13: VEH prototype attached to a top lateral (TL) bracing on the Forth Road Bridge.

significant vibration. Figures 14 and 15 illustrate excerpts of the vibration profile measured by a $16 \mathrm{~g} 3-$ axes accelerometer data-logger ( $800 \mathrm{~Hz}$ sampling rate) from the top lateral. FFT (fast Fourier transform) and STFT (short-time Fourier transform) were computed from the time domain raw data in MATLAB. A 256-point Chebyshev window was employed for the STFT. The value for the window function was manually adjusted to yield the reasonable resolution.
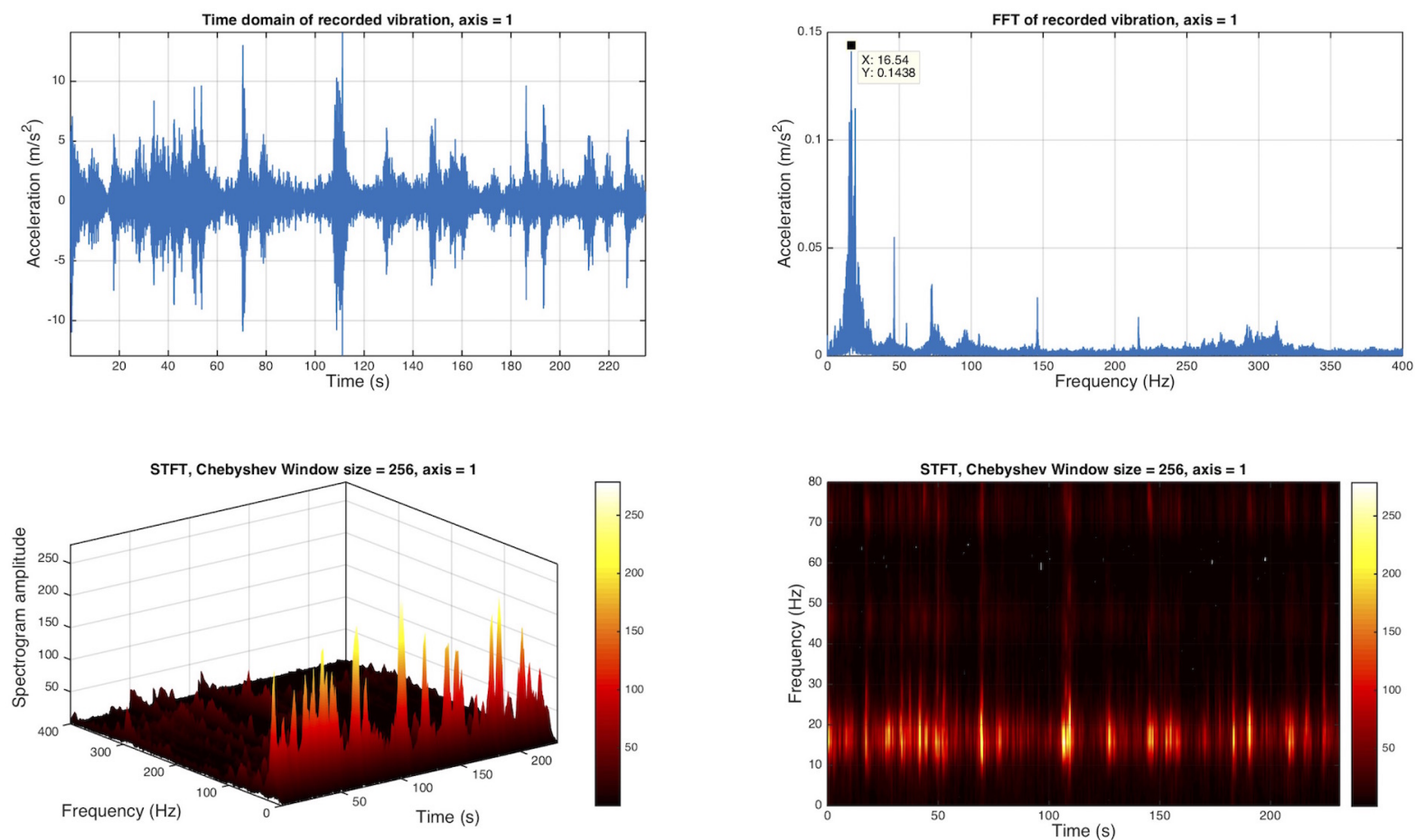

Figure 14: Excerpts of vibration profile recorded from a top lateral bracing of the Forth Road Bridge. Accelerometer attached to the side of the beam, along the vertical axis.

It can be noted that relatively notable vibration amplitude can be seen between $\sim 10 \mathrm{~Hz}$ to $\sim 30 \mathrm{~Hz}$ when attached to the side of the top lateral and between $\sim 7 \mathrm{~Hz}$ and $\sim 26 \mathrm{~Hz}$ when attached to the top of the beam. These are localised structural vibration of the top lateral bracing beam, induced by passing traffic above deck. While both measurements are for the same localised region of the structure, the measurement recorded for the top mount illustrated a significantly larger frequency-specific amplitude than side mount. However, a part of the magnitude difference was also contributed by the type of the traffic passing by at the time of the measurement. For the top lateral bracing, only two axes (one vertical and one horizontal) demonstrated significant vibration amplitude, while the axis along the length of the beam recorded relatively low vibration due to the much higher stiffness in the long axis.

From the STFT plots, the time-varying nature of the frequency peaks can be observed. This demonstrates the intermittent nature of the bridge vibration. Additionally, the frequency response itself varies 

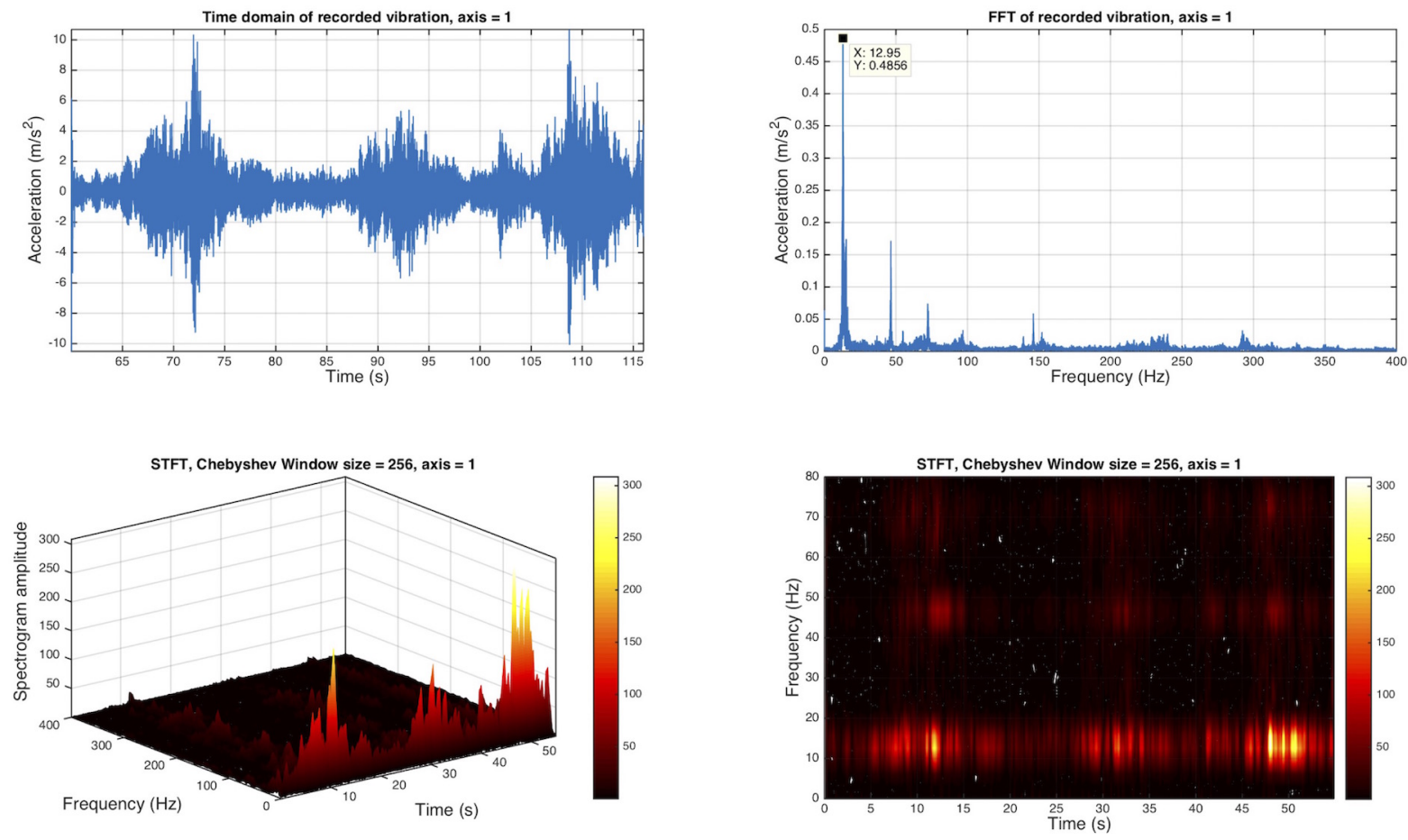

Figure 15: Excerpts of vibration profile recorded from a top lateral bracing of the Forth Road Bridge. Accelerometer attached to the top of the beam, along the horizontal (breadth) axis.

with time. This can be especially challenging for resonant-based VEH, as the resonator might be primarily operating in the transient resonant build up state and the resonant peak cannot be achieved with the fast-varying nature of the frequency source.

During the trial test, the vibration from the cross girder (vertical axis) and the 2 axes (horizontal and vertical) on the top lateral, was able to charge up the $\sim 5 \mathrm{mF}$ super capacitor by the harvester prototype and supplied a regulated $2.5 \mathrm{~V}_{d c}$ source to the WSN mote via the power conditioning circuit. Successful communication with a receiver mote from the purely vibration powered transmitter was successfully recorded during the trial test.

With a transmission rate of once a minute, the power drain was replenished by the vibration input, and a net-positive-power was observed by the vibration powered WSN mote at both the cross girder and the top lateral. The top lateral location provided a faster energy build up across the storage capacitor due to slightly larger vibration amplitude.

The approximate estimated average net-power consumption of the VEH-powered WSN mote are: cross girder vertical axis $+20 \mu \mathrm{W}$, top lateral vertical axis $+160 \mu \mathrm{W}$ and top lateral horizontal axis +300 $\mu \mathrm{W}$. These average power estimates $P_{a v}$ were calculated based on the voltage build-up from $V_{c a p 1}$ to $V_{\text {cap } 2}$ across the storage capacitor with capacitance $C_{c a p}$ for a given period of time $T$ (equation 3 ).

$$
P_{a v}=\frac{1}{2 T} C_{c a p}\left(V_{c a p 2}^{2}-V_{c a p 1}^{2}\right)
$$

These power values take into account the power used by the WSN mote, loss at the conditioning circuitry and impedance mis-match. Therefore, these relate to the net conditioned power gain. The average raw $\mathrm{AC}$ power generated by the harvester were estimated to be at higher levels. A summary is presented in table 2. The conversion efficiency for the conditioned power compared to load-matched raw AC power, employed for the calculation here, were based on pre-calculated experimental values in the lab (20\% to $30 \%$ for the voltage range of $\mathrm{V}_{\text {cap }}$ explored here: $3.5 \mathrm{~V}$ to $5.0 \mathrm{~V}$ ).

During a specified time period $T$, there would be instantaneous power peaks that are a lot higher than $P_{a v}$ as well as regions of very low or null power. Therefore, as long as $T$ is sufficiently large enough to encompass various representative events, $P_{a v}$ thus derived would provide an approximation to calculate the amount of extractable and useable energy $E=P_{a v} t$. 
Table 2: The estimated average power values generated by the harvester prototype at various locations on the Forth Road Bridge. Typical traffic conditions were assumed for the day of measurement.

\begin{tabular}{cc|ccc}
$\begin{array}{c}\text { Location and } \\
\text { orientation }\end{array}$ & $\begin{array}{c}\text { Active frequency } \\
\text { range }(\mathrm{Hz})\end{array}$ & $\begin{array}{c}\text { Raw AC power } \\
(\mu \mathrm{W})\end{array}$ & $\begin{array}{c}\text { Conditioned power } \\
(\mu \mathrm{W})\end{array}$ & $\begin{array}{c}\text { Net excess power } \\
(\mu \mathrm{W})\end{array}$ \\
\hline Cross girder vertical & 10 to 30 & 160 & 32 & +20 \\
Top lateral vertical & 10 to 30 & 800 & 174 & +160 \\
Top lateral horizontal & 7 to 26 & 1050 & 315 & +300
\end{tabular}

\section{Experimentally-simulated lab test}

Due to the practical difficulty to monitor the various performance parameters of the harvester, power conditioning circuit and the WSN systems, lab-based tests based on the measured vibration data were carried out to demonstrate the operation of the VEH-enabled WSN system. The measured vibration profile from the Forth Road Bridge was used to program the vibration shaker in the lab, in order to experimentally simulate the bridge vibration conditions. Waveform Editor by Agilent Technologies was used to import the measured data and communicate with an Agilent function generator. An accelerometer (ADXL325) was used to characterise the vibration generated by the shaker. The experimentally-simulated vibration was matched to just below the amplitude of the measured vibration acceleration amplitude. Moreover, restrictions of the experimental setup only allowed single axis excitation of the prototype, unlike the multi-axes vibration present in the real site. Therefore, all power results are conservative estimates of the actual achievable power in the real site.
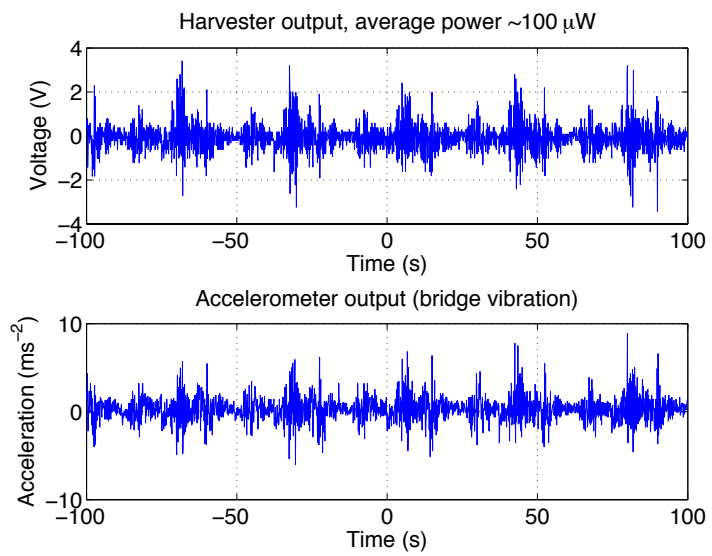

Figure 16: Harvester output from measured bridge vibration data-programmed shaker in the lab, with average power $\sim 100 \mu \mathrm{W}$, active range (when traffic passes by) $\sim 0.5 \mathrm{~mW}$ and peak instantaneous power $\sim 3.3 \mathrm{~mW}$.

Figure 16 illustrates a representative harvester output when subjected to a shaker programmed to the vibration profile measured from a top lateral bracing at the Forth Road Bridge. In this particular example, the average power across the spectrum is $100 \mu \mathrm{W}$ (see table 2 for measurements from the site test), the average power output for one of the vibration active range (when traffic is passing by) is $\sim 0.5$ $\mathrm{mW}$ and the peak instantaneous power recorded was $3.3 \mathrm{~mW}$. These power values are raw AC power produced by the harvester prototype.

While the lab-based power output values were intended to be conservative, this value is about ten times less than the real site recoverable power recorded in table 2. One possible explanation for the significantly lower power output in the lab environment could be a result of the limitation to a single axis vibration during the lab-based tests. Whereas in the site environment, vibration from multiple axes can be coupled in simultaneously, promoting the chance of entering into parametric resonant operation more readily.

The shaker vibration was continuously looped and used to assess various WSN and sensor systems in the lab. Figure 17 is a load tuned $\left(220 \mathrm{k} \Omega\right.$ ) scenario across a regulated $2.5 \mathrm{~V}_{d c}$ output from the power conditioning circuit. The harvester is subjected to the measured vibration data-programmed shaker, while the supercapacitor voltage is monitored over time. Each period of the voltage cycle shown in $\mathrm{V}_{\text {cap }}$ in figure 17 relates to one loop of vibration data. Various load resistance was varied until the average supercapacitor voltage (each cycle/loop) maintains over time. Thus, this is when the power drain by the 

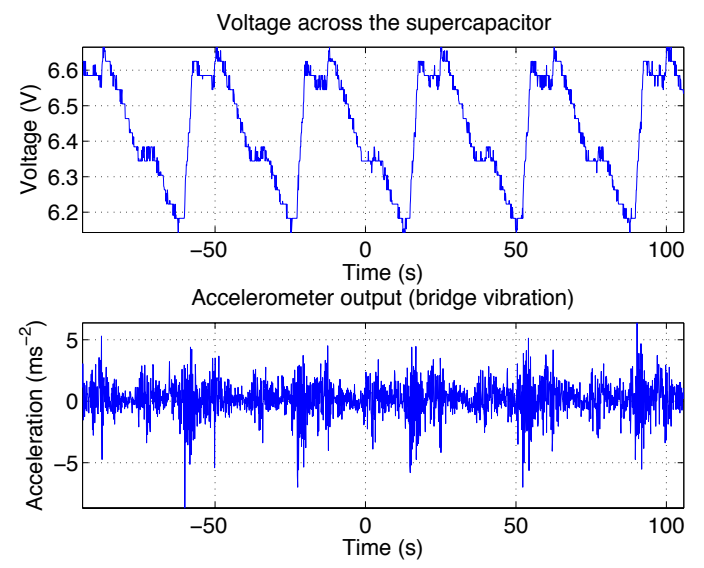

Figure 17: Conditioned power output from the prototype harvester when subjected to measured bridge vibration data-programmed shaker in the lab.

resistor $(28.4 \mu \mathrm{W})$ equates the conditioned power delivered from the power conditioning circuit. Given a supply of $\sim 100 \mu \mathrm{W}$ average raw AC power from the harvester as determined above, the efficiency from the power conditioning board was under a third.
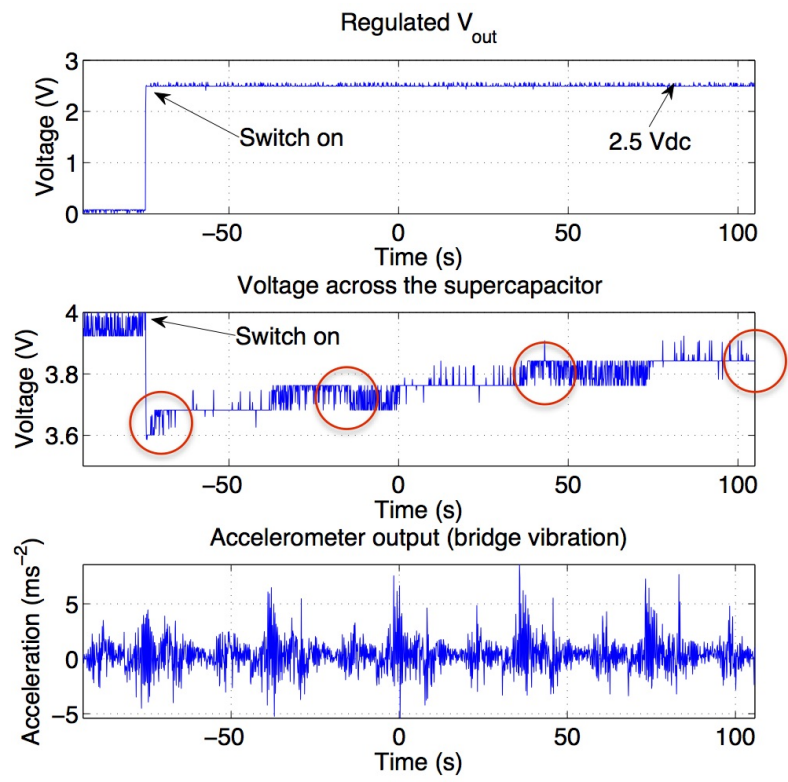

Figure 18: Harvester powered (by shaker programmed with measured bridge vibration) wireless sensor mote via a power conditioning board. First wireless transmission, including power drain required for start-up and routing, takes place upon switch on. Succeeding transmissions takes place once per minute. 4 successful transmissions took place in the displayed time range (circled in in the voltage profile of the supercapacitor). Net power drain is compensated (in excess) by the conditioned harvested power, as the voltage across the supercapacitor slowly but steadily rises.

Therefore, in this scenario, over two thirds of power were not recovered by the power conditioning circuit. The conversion efficiency, determined from conditioned power by load-matched raw AC power, ranges in the order of $\sim 20 \%$ when $\mathrm{V}_{\text {cap }}$ is in the range of $3.5 \mathrm{~V}$ to $4.5 \mathrm{~V}, \sim 30 \%$ when $\mathrm{V}_{\text {cap }}$ is in the range of $5.0 \mathrm{~V}$ to $6.0 \mathrm{~V}$ and $\sim 40 \%$ when $\mathrm{V}_{\text {cap }}$ is in the range of $7.0 \mathrm{~V}$ to $9.0 \mathrm{~V}$.

Figure 18 demonstrates the harvester subjected to the bridge vibration data-programmed shaker, powering up a WSN mote via the power conditioning circuit. Upon achieving $4.0 \mathrm{~V}$ across the supercapacitor, the $2.5 \mathrm{~V}$ regulated DC output is switched on for the wireless mote. Upon initial turn on, the mote drains $0.4 \mathrm{~V}$ across a $\sim 5 \mathrm{mF}(\sim 7.6 \mathrm{~mJ})$ storage capacitor for starting up the micro-controller, wireless routing and 1 package of transmission. Subsequent transmissions takes place once every minute and drains $\sim 0.03 \mathrm{~V}$ in the displayed range $(\sim 0.5 \mathrm{~mJ})$. The average power drain is less than the replenished 
conditioned power, as the voltage across the supercapacitor continues to rise over time.

\section{Industrial Compressor Engine Demonstration}

Apart from civil infrastructure, structural health monitoring (SHM) is also of interest for vehicles, aircrafts, ships, power plant, industrial processing, distribution systems as well as various engine and turbine monitoring applications (Chang, 2003). VEH enabled SHM is suitable for environments with notable levels of vibration such as vibrating motors for engines or compressors. It has been previously demonstrated on a ferry engine (Weddell et al., 2012). Unlike civil infrastructure, the vibration of engines and compressors are typically fixed frequency sources, corresponding to the load and RPM (revolutions per minute) of the engine. While load and speed variations would induce a shift in frequency, it is relatively less time-varying. Whereas, vibration from sources such as rail tracks (Jia et al., 2013c) and bridges can be intermittent, fast time-varying (frequency) and span across a relatively broader frequency bandwidth.

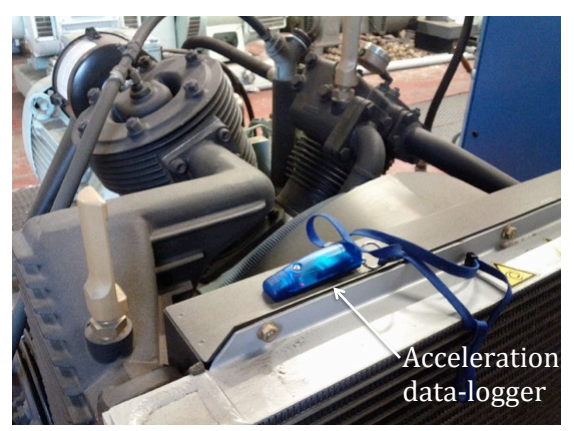

Figure 19: A reciprocal compressor in the basement of the Inglis building of the Department of Engineering, University of Cambridge.

In this paper, an example of industrial engine monitoring is presented for the auto-parametric VEH prototype. The subject chosen was a reciprocal air compressor ( 1400 RPM) suppling a supersonic tunnel in the Cambridge University Engineering Department (CUED). Figure 19 shows the compressor in the basement of the Inglis building of the CUED. Vibration was measured from the support frame of the compressor, rather than from the casing of the compressor itself.

The compressor intermittently switches on throughout the working day when the supersonic tunnel is in use. The typical continuous operational period of the compressor ranges from tens of minutes to $>1$ hour. Currently the operational status of the compressors in the engine room are manually and periodically monitored by the technician staff. However, the acoustic noise levels in the engine room can potentially pose a health risk to the monitoring staff, despite the use of protective gear.

Figure 20 illustrates an excerpt of the measured vibration profile of the reciprocal compressor in the vertical axis. Only one of the two horizontal axes and the vertical axis demonstrated significant vibration amplitude; where parallels can be drawn with the bridge bridge. For the engine, this could be a result of the centripetal force exerted by the rotating motor in a single 2-dimensional plane.

A first frequency peak can be observed at $\sim 25 \mathrm{~Hz}$, which approximately corresponds to the RPM of the engine. Additional peaks, albeit at smaller magnitude, can be observed at regular integer intervals of the fundamental frequency; thus, suggesting the presence of harmonic components. From STFT, it can be seen that the fundamental frequency peak is relatively time-invariant in terms of both the average amplitude and the active frequency range. This provides a good source for resonant-based VEH.

Figure 21 illustrates the experimentally-simulated response of the VEH prototype when subjected to shaker vibration programmed by the measured compressor data. An average of $\sim 540 \mu \mathrm{W}$ raw AC power output was recorded. Same as the bridge scenario in section III, the programmed vibration amplitude was just below the real vibration. Coupled with solely a single axis excitation, this would be a relatively conservative value than the vibration available in the real situation (in the bridge case, lab result yielded 10 times less power than real site measurement). However, real site testing on the compressor, using a VEH prototype, has not been carried out yet due to current limitations with health and safety regulations for uncertified equipment in an operational engine room.

Nonetheless, the experimentally-simulated vibration in the lab was more than sufficient to power up the WSN system with $\sim 540 \mu \mathrm{W}$ of raw AC power and $\sim 190 \mu \mathrm{W}$ of conditioned power. As can be seen from figure 22, the VEH supply significantly exceeds the power drain by the WSN transmission duty 

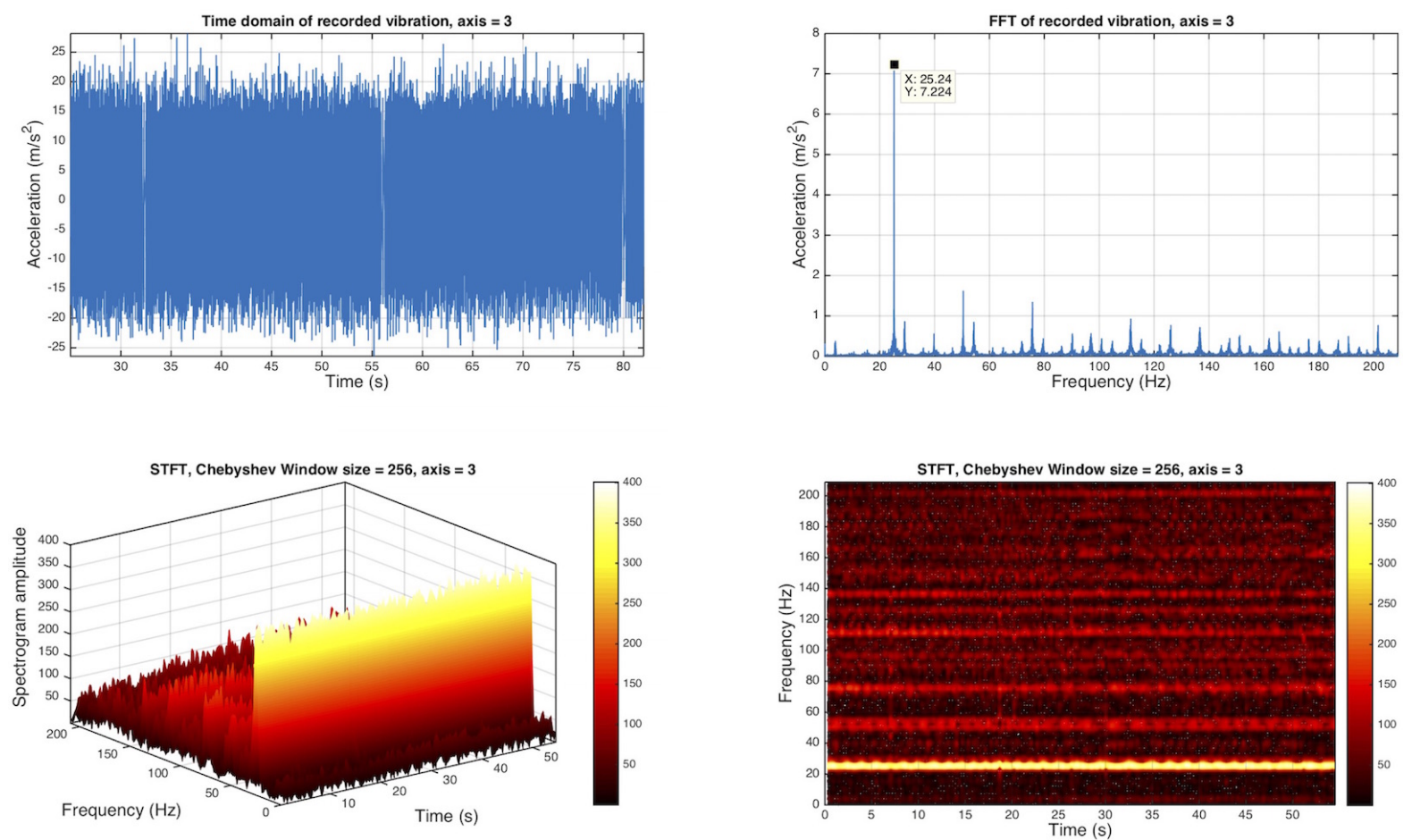

Figure 20: Vibration measured from the top of the frame of a compressor motor in the z-axis (vertical motion).

cycled to once per minute (average consumption of $\sim 11 \mu \mathrm{W}$ ), as $\mathrm{V}_{\text {cap }}$ rapidly increases. Across the time span shown in the figure, 2 successful wireless transmissions took place including the power drain of the mote initialisation. Based on these power budget figures, this particular setup and environment can sustain the same End-Device mote with a transmission rate of up to once every 3 seconds.

\section{Automotive Demonstration}

There has been a growing interest to integrate and/or increase structural health monitoring of various locations in an automotive vehicle (Chang, 2003), which is partially fuelled by automotive regulations such as the implementation of tyre pressure monitoring systems in both the USA and EU (Rezulteo, 2015). The use of ZigBee-based WSN or RFID to either complement or replace wired sensors for intracar communication in the automotive application was demonstrated nearly a decade ago (Tonguz et al., 2006; Tasi et al., 2007). Since then, major automotive companies, such as Volvo (Basso, 2009) and Volkswagen (D'Orazio et al., 2011), have invested in the research and development of wireless sensors to
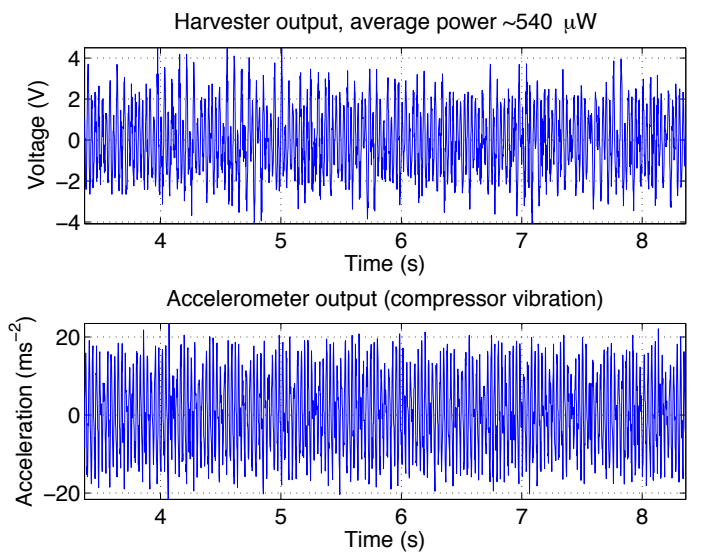

Figure 21: Harvester output from measured compressor vibration data-programmed shaker in the lab. 

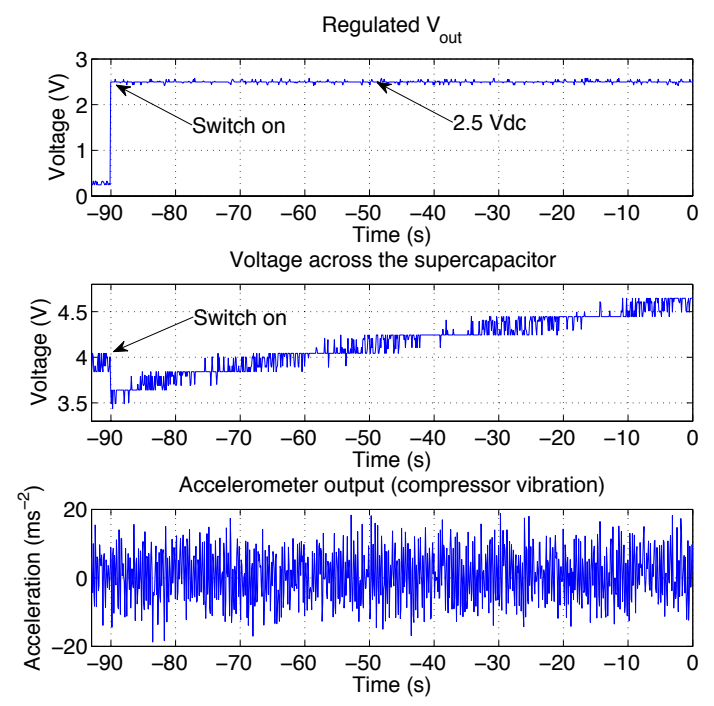

Figure 22: Harvester powered (by shaker programmed with measured compressor vibration) wireless sensor mote via a power conditioning board. First wireless transmission, including power drain required for start up and routing, takes place upon switch on. Succeeding transmissions takes place once per minute. 2 successful transmissions took place in the displayed time domain (1st at $-90 \mathrm{~s}$ and 2nd at $-30 \mathrm{~s}$ ). Net power drain is compensated (in notable excess) by the conditioned harvested power, as the voltage across the supercapacitor rapidly and steadily rises.

replace existing wired solutions for road vehicles.

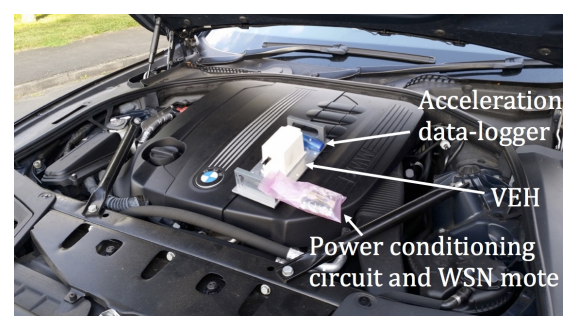

Figure 23: An example location (under the bonnet) on a car where the VEH-powered WSN was tested.

Some of the main motivations for intra-car WSN include,

- There are an increasing number of mandated and/or recommended intelligent monitoring systems and autonomous safety systems for modern automobiles, which would require either wired or wireless sensor systems (D'Orazio et al., 2011).

- Additional mass of the cabling of wired sensors can weigh up to the order of $50 \mathrm{~kg}$ (Tonguz et al., 2006) and extend up to $8 \mathrm{~km}$ in total length (Tavares et al., 2008).

- Minimising cabling can reduce production and fitting cost associated with wiring, improve fuel efficiency in the range of an estimated 5\% (Lee et al., 2009) and reduce the complexity and diagnostic cost for servicing the hundreds of physical interconnections for the wired solution (D'Orazio et al., 2011).

- Due to the limitation of weight, length and complexity of interconnections, wired solutions are not as scalable as their WSN counterparts.

- Cabling are not suitable for moving and/or less accessible locations such as tyres and pipes (D'Orazio et al., 2011; Tavares et al., 2008).

To assess the performance and preliminarily demonstrate the concept of VEH-powered WSN solution in an automobile, both real vehicle testing and lab-based experimentation using measured vibration data 
were carried out. The car employed for the testing was a Bayerische Motoren Werke 525d SE 2011 model, 215 brake horsepower at 4400 RPM, 8-speed automatic gearbox and turbodiesel engine with 6 cylinders (3.0 litres). Various locations in the car, including under the bonnet (beside or above the engine vibration dampener hood) as illustrated in figure 23, beneath the boot (trunk), interior of the vehicle and section of the chassis above the wheel (figure 24).

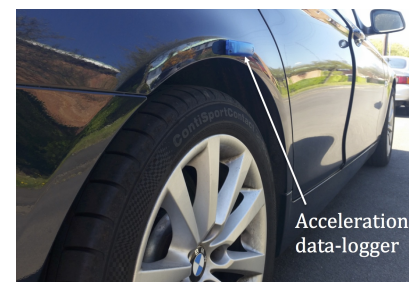

Figure 24: Vibration measured from the chassis above the right back wheel.
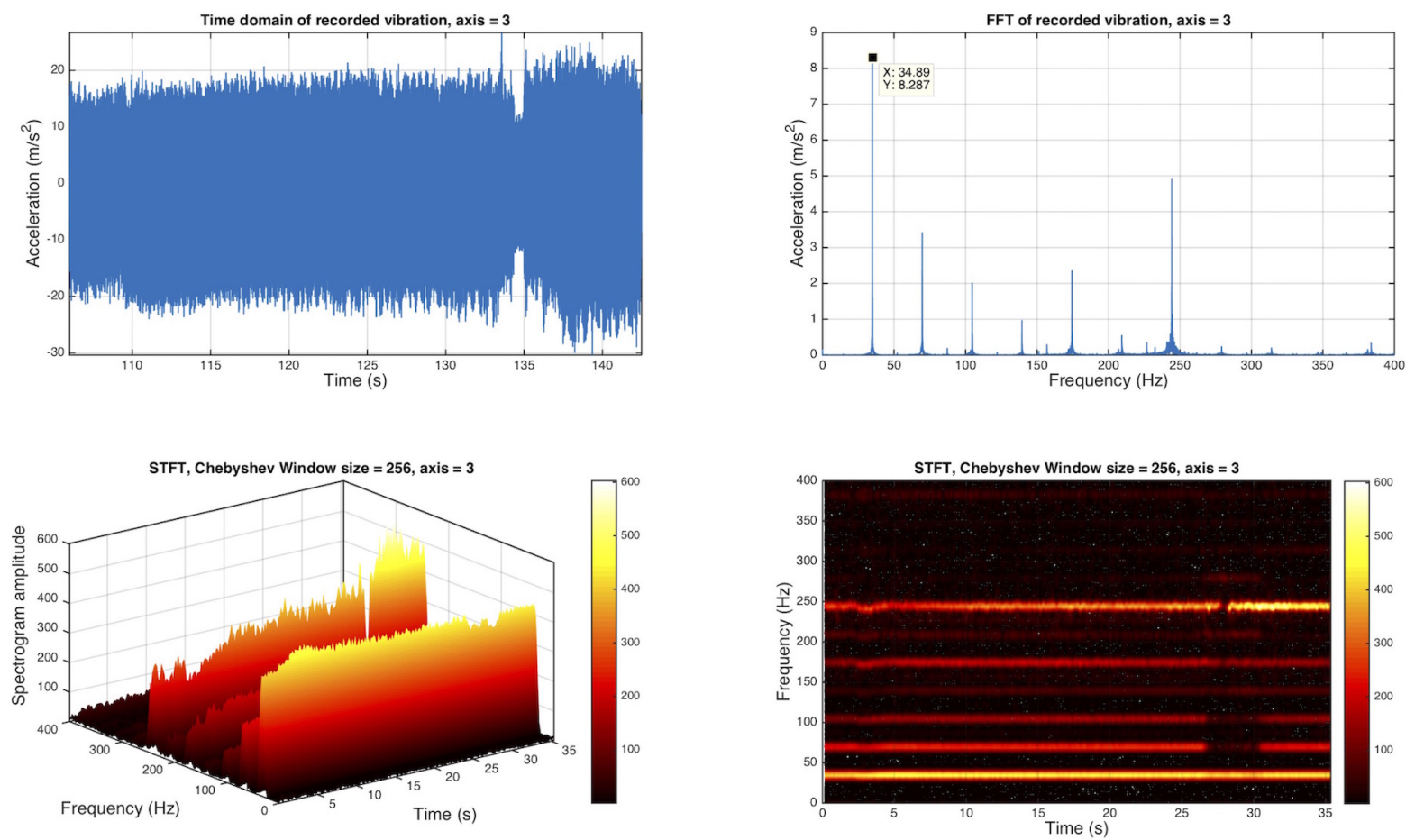

Figure 25: An excerpt of vibration in the z-axis, measured from the top of the vibration damper enclosure of a BMW 525d engine.

An excerpt of measured vibration from above the vibration dampener hood of the vehicle engine is shown in figure 25. It can be seen that a first frequency peak at $\sim 35 \mathrm{~Hz}$ can be seen. This corresponds to the RPM of the engine, which is in the range of $\sim 2100$ RPM. Therefore, as the RPM of the engine varies, this frequency peak also shifts. Vibration elsewhere in the car are generally more responsive to road interface induced excitations ranging from a few hertz to a few tens of hertz. However, for chassis near the wheel, both broadband response mixed with fixed frequencies were seen.

The macro prototype in its current form was not designed for the car vibration and the resonant frequencies (neither fundamental direct mode nor parametric instability region) were not tuned for the vehicle vibration profile. Additionally, various parts of the car was designed with vibration dampener structures to minimise the vibration subjected to the passengers and interior chassis of the car was not assessed during the testings. Therefore, the VEH prototype was subjected to relatively moderate amplitude and frequency mismatched conditions.

For real testing under the bonnet of the car, $\sim 250 \mu \mathrm{W}$ raw AC power and $\sim 51 \mu \mathrm{W}$ conditioned power were measured. Despite the non-resonant operation of the VEH prototype, the generated power was already capable of sustaining the WSN mote duty cycled to 1 transmission per minute (with $\sim 40$ $\mu \mathrm{W}$ excess power). 

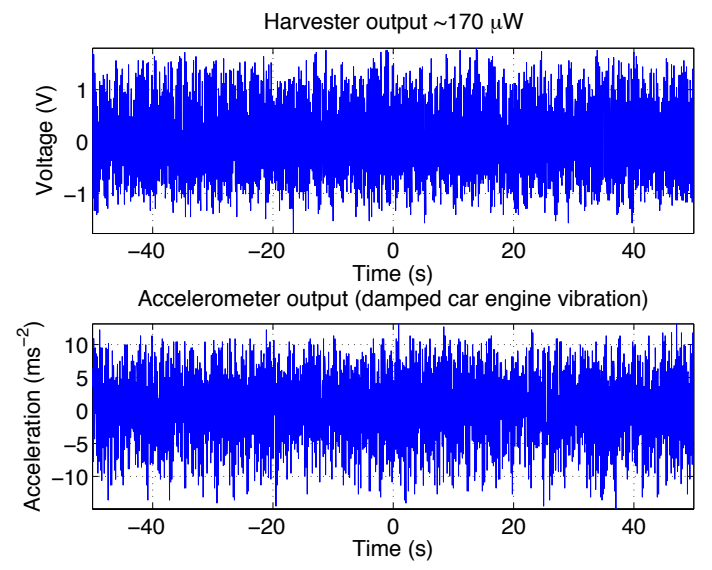

Figure 26: Harvester output from measured damped car engine vibration data-programmed shaker in the lab. The primary frequency of the vibration source $(\sim 35 \mathrm{~Hz})$ does not match the harvester frequencies $(\sim 13 \mathrm{~Hz}$ and $\sim 25 \mathrm{~Hz}$ ). Therefore, the output are primarily non-resonant response.

In the lab environment using experimentally-simulated vibration (conservative conditions and estimates) illustrated in figure $26, \sim 170 \mu \mathrm{W}$ raw AC power and $\sim 34 \mu \mathrm{W}$ conditioned power were generated to power the WSN mote and sensor systems. The VEH-powered WSN mote was tested with this vibration profile for over an hour with a receiver mote connected to a computer and packets recorded on CoolTerm. All packets were successfully received during the test period, without any errors or failed transmissions. Meanwhile during the one-hour test, $\mathrm{V}_{\text {cap }}$ slowly but steadily increased in voltage, demonstrating a net positive power budget. This demonstrated, albeit in a lab environment, the continuous short term stability of the vibration powered wireless system, using a representative vibration source.

\section{VEH-powered sensors and power budget}

In addition to a WSN mote, the harvester prototype powered by the experimentally-simulated bridge vibration was demonstrated to power up and sustain the operation of various low-power sensors. A collection of commercial MEMS accelerometers from Analog Devices were tested (figure 27). This included: ADXL326 $( \pm 16 \mathrm{~g}, 57 \mathrm{mV} / \mathrm{g})$, ADXL325 $( \pm 5 \mathrm{~g}, 174 \mathrm{mV} / \mathrm{g})$, ADXL337 $( \pm 3 \mathrm{~g}, 300 \mathrm{mV} / \mathrm{g})$ and ADXL327 $( \pm 2 \mathrm{~g}, 420 \mathrm{mV} / \mathrm{g})$. The continuous power consumption of these chips at maximum sensor resolution were experimentally measured to be in the range of $\sim 0.5 \mathrm{~mW}$. The regulated DC voltage from the power conditioning board was used as the power supply for the accelerometers.

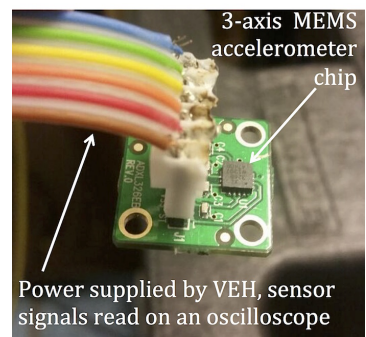

Figure 27: Photograph of a MEMS accelerometer (ADXL326) powered by VEH.

Figure 28 illustrates an example of the MEMS accelerometer powered up by the VEH, via the LTC3588-1 power conditioning board, using the experimentally-simulated Forth Road Bridge vibration. Upon attaining $4.0 \mathrm{~V}$ across the storage capacitor, a $2.5 \mathrm{~V}_{d c}$ was supplied to the accelerometer. The accelerometer in the meanwhile was manually shaken to demonstrate its operational status. While a net drain in power is seen, the available energy budget in this unfavourable situation is still able to sustain the sensor operation for over half a minute. Furthermore, in realistic scenarios, the sensor operation and sensor resolution can be duty-cycled (Analog Devices, 2015) to potentially reduce the average power consumption to the range of a few micro-watts. 

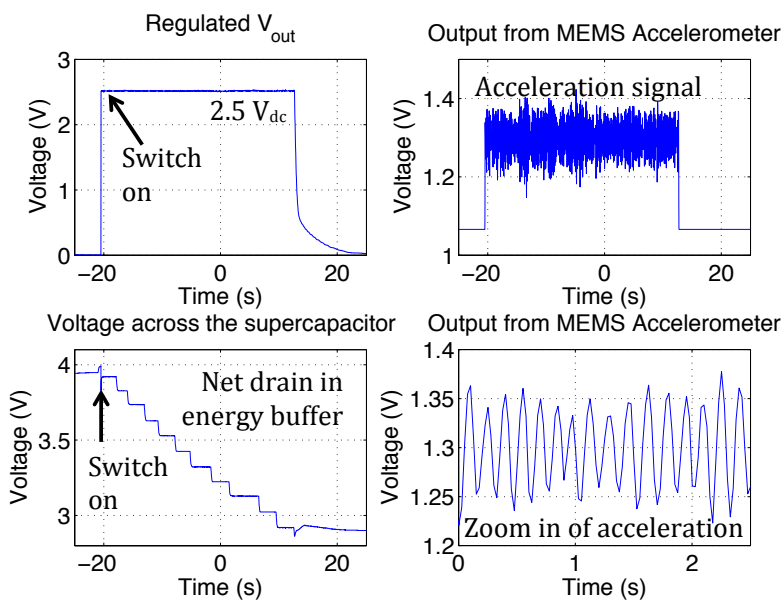

Figure 28: Harvester powered (by shaker programmed with measured bridge vibration) MEMS accelerometer (Analog Devices ADXL326, $\pm 16 \mathrm{~g} 3$-axes) via a power conditioning board. Z-axis sensor output is shown here, as measured by an oscilloscope. A net power drain of $\sim 0.5 \mathrm{~mW}$ was observed over time. However, the energy buffer charged up by the VEH was able to sustain a stable sensor operation for over $30 \mathrm{~s}$.

Further sensors trialled with the VEH prototype driven using measured vibration include: temperature sensor (DS18B20 programmable resolution 1-wire digital thermometer), humidity sensor (Honeywell HIH-4000 series), displacement sensor (potentiometric linear position transducer) and luminosity sensor (light dependent resistor). Table 3 and table 4 detail the available power budget achievable by the current VEH prototype and power demand of the various sensors (continuous/duty-cycled).

Table 3: Average power recorded for the current prototype in various environments. Site refers to measured values in real site environments, while lab refers to conservative lab tests using measured vibration data from representative environments. Note, the power values are average over a given period of time including (typically between 1 and 10 minutes, depending on variability). For intermittent vibration environments, both active and inactive events are included. Instantaneous power or average power of active events can attain higher values.

\begin{tabular}{|c|c|c|c|c|}
\hline \multirow{2}{*}{ Environment } & \multicolumn{2}{|c|}{ Power output at site } & \multicolumn{2}{|c|}{ Power output in lab } \\
\hline & Raw $(\mu \mathrm{W})$ & Conditioned $(\mu \mathrm{W})$ & $\operatorname{Raw}(\mu \mathrm{W})$ & Conditioned $(\mu \mathrm{W})$ \\
\hline FRB top lateral & 1050 & 315 & 100 & 28.4 \\
\hline Compressor & $\mathrm{n} / \mathrm{a}$ & $\mathrm{n} / \mathrm{a}$ & 540 & 190 \\
\hline BMW near engine & 250 & 51 & 170 & 34 \\
\hline
\end{tabular}

It should be noted that the current VEH prototype was only tuned (during design) for the bridge application. Therefore, the car application experienced only non-resonant/reduced power output for this particular iteration. If designed specifically to match the active vibration range of the automotive application, much higher power output can be achieved. Additionally, site values indicate measurements in real environment, and conservative values represent experimentally-simulated lab measurements.

\section{Conclusion and future work}

This paper presented a packaged electromagnetic vibration energy harvester capable of operation in both directly excited resonance and auto-parametrically excited resonance. With a practical operational volume of $126 \mathrm{~cm}^{-3}$, the prototype is able to deliver a peak of $78.9 \mathrm{~mW}$ and $4.5 \mathrm{~Hz}-3 \mathrm{~dB}$ bandwidth at parametric resonance when driven at $23.5 \mathrm{~Hz}$ and $1 \mathrm{~g}_{r m s}$. Additionally, real site and representative environment testing of the harvester recorded average power output up to $\sim 1 \mathrm{~mW}$ from a top lateral bracing on Forth Road Bridge (a $2.5 \mathrm{~km}$ suspension bridge in Scotland), $\sim 0.5 \mathrm{~mW}$ from a compressor engine and $\sim 0.25 \mathrm{~mW}$ (non-resonant response) from under the bonnet of a car.

A power conditioning circuit was used to convert the raw AC power from the harvester into a stable DC source for a wireless sensor mote. With duty-cycling of one transmission per minute and ultra-low power operation protocol, the average power of a wireless mote (without accounting for sensor usage) was reduced to $\sim 11 \mu \mathrm{W}$. Therefore, successful wireless communication was established between a harvesterpowered mote and a battery-powered mote at both real site trial and representative environment testing 
Table 4: Sensors that were powered up and demonstrated by the VEH prototype, along with the associated power budget of these sensors. Note, the power budget values are average power consumption over the period of each sampling cycle. Instantaneous power can be at a higher value.

\begin{tabular}{|c|c|c|}
\hline Sensor & Power budget & Comments \\
\hline \multicolumn{3}{|c|}{ continuous sensor operation } \\
\hline MEMS strain gauge & $\sim 80(\mu \mathrm{W})$ & $\begin{array}{l}\text { In-house sensor (Jia et al., } \\
\text { 2015a), with loading loss due to }\end{array}$ \\
\hline MEMS strain gauge & $\sim 2(\mu \mathrm{W})$ & $\begin{array}{l}\text { cabling } \\
\text { In-house sensor (Jia et al., }\end{array}$ \\
\hline & & $\begin{array}{l}\text { 2015a), without loading loss } \\
\text { due to cabling }\end{array}$ \\
\hline MEMS accelerometer & $\sim 500(\mu \mathrm{W})$ & Anlog Devices ADXL series \\
\hline \multicolumn{3}{|c|}{ duty-cycled to one sampling per minute } \\
\hline MEMS accelerometer & $\sim 3(\mu \mathrm{W})$ to $\sim 10(\mu \mathrm{W})$ & Anlog Devices ADXL series \\
\hline Digital thermometer & $\sim 18(\mu \mathrm{W})$ & Maxim Integrated DS18B20 \\
\hline Potentiometric displacement & $\sim 150(\mu \mathrm{W})$ & In-house linear potentiometer \\
\hline Light dependent resistor & $\sim 250(\mu \mathrm{W})$ & $820 \mathrm{k} \Omega$ \\
\hline Humidity sensor & $\sim 160(\mu \mathrm{W})$ & Honeywell HIH-4000 series \\
\hline
\end{tabular}

in the lab. Low power sensors such as MEMS accelerometers, etc. were also demonstrated with the harvester system using representative vibration profiles.

Future and ongoing work includes development of dedicated power conditioning circuitry in order to maximise the conversion efficiency from raw AC power into regulated DC power. Some of the primary challenges include impedance matching and overcoming the diode voltage thresholds. Furthermore, the macro-scale harvesters will be further developed to enhance its robustness and reliability in order to realise a long term real site deployment trial. This would enable a longitudinal study into the performance of a harvester-powered wireless sensor system in a real world structural health monitoring environment.

\section{Acknowledgement}

This work was supported by EPSRC (grant: EP/L010917/1) and the Cambridge Centre for Smart Infrastructure and Construction. Special thanks to Barry Colford and Keith Perryman at Forth Road Bridge for providing access and support to the site testing.

\section{Author contributions}

- YJ, JY, KS and AAS: Conceived the research and the early work on parametrically excited VEH.

- YJ and AAS: Designed the prototype, developed the models and analysed the results.

- YJ, JY, SD, TF and AAS: Designed and/or undertook experimentation of VEH, WSN and sensors.

- YJ, PF, CM and AAS: Designed and/or engaged in work associated with Forth Road Bridge.

- YJ: Designed and undertook work on automobile and compressor; and authored the manuscript.

- All authors reviewed the manuscript.

\section{Competing financial interests}

Yu Jia, Jize Yan, Kenichi Soga and Ashwin A. Seshia are co-founders of 8power Ltd.

\section{References}

Amey (2017) Forth road bridge. URL https://www.forthroadbridge.org. 
Analog Devices (2015) 3-axis adxl345 series digital accelerometer. URL http://www . analog.com/media/ en/technical-documentation/data-sheets/ADXL345.pdf.

Andosca R, McDonald TG, Genova V, Rosenberg S, Keating J, Benedixen C and Wu J (2012) Experimental and theoretical studies on mems piezoelectric vibrational energy harvesters with mass loading. Sens. Actuators A 178: 76-87.

Arroyo E and Badel A (2011) Electromagnetic vibration energy harvesting device optimization by synchronous energy extraction. Sensors and Actuators A: Physical 171(2): $266-273$.

Atmel Corporation (2015) Atmel avr2130: Lightweight mesh developer guide, application note. URL http://www. atmel.com/tools/LIGHTWEIGHT_MESH. aspx?tab=documents.

Basso R (2009) Wireless sensor networks in a vechile environment. Masters thesis, Chalmers University of Technology, Sweden.

Beeby S, Tudor M and White N (2006) Energy harvesting vibration sources for microsystems applications. Meas. Sci. Technol. 17(12): R175-R195.

Beeby S and White N (2010) Energy Harvesting for Autonomous Systems. USA: Artech House.

Burrow S and Clare L (2007) A resonant generator with non-linear compliance for energy harvesting in high vibrational environments. In: Proceedings of the IEEE International Electric Machines and Drives Conference. Antalya, pp. 715-720.

Cervenka V, Mraz L and Komosny D (2014) Comprehensive performance analysis of lightweight mesh and its comparison with zigbee pro technology. Wirel. Pers. Commun. 78(2): 1527-1538.

Challa V, Prasad M and Fisher F (2011) Towards an autonomous self-tuning vibration energy harvesting device for wireless sensor network applications. Smart Mater. Struct. 20(2): 025004 (11pp).

Chang FK (ed.) (2003) Structural Health Monitoring: from diagonstics and Prognostrics to structural health management. Lancaster, Pennsylvania, USA: DEStech Publications, Inc.

D'Orazio L, Visintainer F and Darin M (2011) Sensor networks on the car: State of the art and future challenges. In: Proc. IEEE DATE. Grenoble, pp. 1-6.

Erturk A and Inman D (2011) Piezoelectric energy harvesting. New Delhi, India: Wiley.

Feng G and Hung $J$ (2007) Optimal fom designed piezoelectric microgenerator with energy harvesting in a wide vibration bandwidth. In: Proceedings of the 2nd IEEE International Conference on Nano/Micro Engineered and Molecular Systems. Bangkok, pp. 511-514.

Galchev TV, McCullagh J, Peterson RL and Najafi K (2011) Harvesting traffic-induced vibrations for structural health monitoring of bridges. Journal of Micromechanics and Microengineering 21(10): 104005 .

Green PL, Papatheou E and Sims ND (2013) Energy harvesting from human motion and bridge vibrations: An evaluation of current nonlinear energy harvesting solutions. Journal of Intelligent Material Systems and Structures 24(12): 1494-1505. DOI:10.1177/1045389X12473379.

Gutierrez JA, Naeve M, Callaway E, Bourgeois M, Mitter V and Heile B (2011) Ieee 802.15.4: A developing standard for low-power, low-cost wireless personal area networks. IEEE Network 15(5): 12-19.

$\mathrm{Hu}$ Y, Xue H and Hu H (2007) A piezoelectric power harvester with adjustable frequency through axial preloads. Smart Mater. Struct. 16(5): 1961-1966.

Jia Y, Do CD, Zou X and Seshia AA (2015a) A hybrid vibration powered microelectromechanical strain gauge,. IEEE Sens. J. 16(1): 235-241.

Jia Y and Seshia A (2013) Directly and parametrically excited bi-stable vibration energy harvester for broadband operation. In: Proc. Transducers 2013, volume DOI: 10.1109/Transducers.2013.6626801. Barcelona, Spain, pp. 454-457.

Jia Y and Seshia AA (2014) An auto-parametrically excited vibration energy harvester. Sens. Actuators A 220: 69-75. 
Jia Y, Yan J, Feng T, Du S, Fidler P, Soga K, Middleton C and Seshia A (2015b) A vibration powered wireless mote on the forth road bridge. In: J. Phys. Conf. Ser., 1, volume 660. p. 5pp.

Jia Y, Yan J, Soga K and Seshia A (2013a) Multi-frequency operation of a mems vibration energy harvester by accessing five orders of parametric resonance. In: J. Phys. Conf. Ser., 1, volume 476. pp. 607-611.

Jia Y, Yan J, Soga K and Seshia A (2013b) Parametrically excited mems vibration energy harvesters with design approaches to overcome initiation threshold amplitude. J. Micromech. Microeng 23(11): 10pp.

Jia Y, Yan J, Soga K and Seshia A (2013c) A parametrically excited vibration energy harvester. J. Intel. Mat. Syst. Str. DOI: 10.1177/1045389X13491637.

Jia Y, Yan J, Soga K and Seshia AA (2014) Parametric resonance for vibration energy harvesting with design techniques to passively reduce the initiation threshold amplitude. Smart Mater. Struct. 23(6): 13.

Kazmierski T and Beeby S (eds.) (2011) Energy Harvesting Systems Principles, Modeling and Applications. Springer US.

Lee H, Tasi HM and Tonguz O (2009) On the security of intra-car wireless sensor networks. In: IEEE VTC. Anchroage, AK, pp. 1-5.

Mann B and Sims N (2009) Energy harvesting from the nonlinear oscillations of magnetic levitation. $J$. Sound Vib. 319(1-2): 515-530.

Mitcheson P, Yeatman E, Rao G, Holmes A and Green T (2008) Energy harvesting from human and machine motion for wireless electronic devices. Proc. IEEE 96(9): 1457-1486.

Montanini R and Quattrocchi A (2016) Experimental characterization of cantilever-type piezoelectric generator operating at resonance for vibration energy harvesting. AIP Conference Proceedings 1740(1): 060003. DOI:10.1063/1.4952675.

Muriuki M (2004) An investigation into the design and control of tunable piezoelectric resonators. PhD Thesis, School of Engineering, University of Pittsburgh.

Peigney M and Siegert D (2013) Piezoelectric energy harvesting from traffic-induced bridge vibrations. Smart Materials and Structures 22(9): 095019.

Perpetuum (2017) Perpetuum - products, pmg-17. URL https://perpetuum.com/technology/.

Petropoulos T, Yeatman E and Mitcheson P (2004) Mems coupled resonators for power generation and sensing. In: Micromechanics Europe. Leuven, Belgium, pp. 261-264.

Priya S and Inman D (2009) Energy Harvsting Technologies. New York: Springer US.

Ramlan R, Brennan M, Mace B and Kovacic I (2010) Potential benefits of a non-linear stiffness in an energy harvesting device. Nonlinear Dynam. 59(4): 545-558.

Rezulteo (2015) Tyre pressure monitoring systems (tpms) are now obligatory. URL http://www.rezulteo-tyres.co.uk/regulations-and-safety/ tyre-pressure-monitoring-systems-tpms-are-now-obligatory-4511.

Shahruz S (2006) Design of mechanical band-pass filters for energy scavenging. J. Sound Vib. 292(3-5): 987-998.

Soliman M, Abdel-Rahman E, El-Saadany E and Mansour R (2008) A wideband vibration-based energy harvester. J. Micromech. Microeng 18(11): 115021 (11pp).

Soliman M, Abdel-Rahman E, El-Saadany E and Mansour R (2009) A design procedure for wideband micropower generators. J. Microelectromech. Syst. 18(6): 1288-1299.

Spreemann D and Manoli Y (2012) Electromagnetic vibration energy harvesting devices: architectures, design, modeling and optimization, Advanced Microelectronics, volume 35. New York: Springer Science and Business Media. 
Stanton S, McGehee C and Mann B (2010) Nonlinear dynamics for broadband energy harvesting: Investigation of a bistable piezoelectric inertial generator. Physica D 239(10): 640-653.

Tasi HM, Saraydar C, Talty T, Ames M, Macdonald A and Tonguz O (2007) Zigbee-based intra-car wireless sensor network. In: IEEE ICC. Glasgow, pp. 3965-3971.

Tavares J, Velaz F and Ferro J (2008) Application of wireless sensor networks to automobiles. Meas. Sci. Technol. 8(3): 65-70.

Tonguz O, Tasi HM, Talty T, Macdonald A and Saraydar C (2006) Rfid technology for intra-car communications: A new paradigm. In: Proc. IEEE VTC. Montreal, Que, pp. 1-6.

Trigona C, Maiorca F, Ando B and Baglio S (2013) Tri-stable behaviour in mechanical oscillators to improve the performance of vibration energy harvesters. In: Transducers. Barcelona, Spain, pp. 458461.

Weddell A, Zhu D, Merrett G, Beeby S and Al-Hashimi B (2012) A practical self-powered sensor system with a tunable vibration energy harvester. In: PowerMEMS. Atlanta, Georgia, USA, pp. 105-108.

Wong Z, Yan J, Soga K and Seshia A (2009) A multi-degree-of-freedom electrostatic mems power harvester. In: PowerMEMS. Washington, pp. 300-303.

Xue H, Hu Y and Wang Q (2008) Broadband piezoelectric energy harvesting devices using multiple bimorphs with different operating frequenciesple bimorphs with different operating frequencies. IEEE Transactions on Ultrasonics, Ferroelectrics and Frequency Control 55(9): 2104-2108.

Zhu D and Beeby S (2013) A coupled bistable structure for broadband vibration energy harvesting. In: Transducers. Barcelona, Spain, pp. 446-449. 